\title{
FBMC System: An Insight into Doubly Dispersive Channel Impact
}

\author{
Lei Zhang, Pei Xiao, Adnan Zafar, Atta ul Quddus and Rahim Tafazolli
}

\begin{abstract}
It has been claimed that the filter bank multicarrier (FBMC) systems suffer from negligible performance loss caused by moderate dispersive channels in the absence of guard time protection between symbols. However, a theoretical and systematic explanation/analysis for the statement is missing in the literature to date. In this paper, based on one-tap minimum mean square error (MMSE) and zero-forcing (ZF) channel equalizations, the impact of doubly dispersive channel on the performance of FBMC systems is analyzed in terms of mean square error (MSE) of received symbols. Based on this analytical framework, we prove that the circular convolution property between symbols and the corresponding channel coefficients in the frequency domain holds loosely with a set of inaccuracies. To facilitate analysis, we first model the FBMC system in a vector/matrix form and derive the estimated symbols as a sum of desired signal, noise, inter-symbol interference (ISI), inter-carrier interference (ICI), inter-block interference (IBI) and estimation bias in the MMSE equalizer. Those terms are derived one-by-one and expressed as a function of channel parameters. The numerical results reveal that in harsh channel conditions, e.g., with large Doppler spread or channel delay spread, the FBMC system performance may be severely deteriorated and error floor will occur.
\end{abstract}

Index Terms-FBMC, IBI, ICI, ISI, one-tap channel equalization, circular convolution, dispersive/distortion channel

\section{INTRODUCTION}

As a promising air-interface candidate solution for the next generation wireless communications, filter bank multicarrier (FBMC) has drawn significant attention by both academia and industry in the last few years [1], [2], [3], [4], [5]. Apart from its main advantage of significantly reduced out-ofband emission in comparison to the widely used orthogonal frequency division multiplexing (OFDM) system [1], [2], [6]; another major benefit is ease in the implementation of multi-user (MU) access in the uplink transmission since the strict synchronization requirements for OFDM systems can be relaxed in FBMC systems [7], [8]. In addition, it has been reported in [9] that FBMC system is more robust to carrier frequency offset (CFO) [10], which is critical for this waveform when employed in high mobility environments and multi-cell cooperation scenarios [7], [11].

Unlike the widely used CP (cyclic-prefix) based OFDM system, where the effect of frequency selective channels can be removed with low complexity one-tap channel equalization. The FBMC system, however, may encounter inter-symbol interference (ISI) and inter-carrier interference (ICI) caused

Copyright (c) 2015 IEEE. Personal use of this material is permitted. However, permission to use this material for any other purposes must be obtained from the IEEE by sending a request to pubs-permissions@ieee.org. The authors are with the Institute for Communication Systems (ICS), University of Surrey, Guildford, GU2 7XH, UK (Email: lei.zhang@surrey.ac.uk). by either imperfect prototype filter or dispersive channels that can not be eliminated completely by low-complexity channel equalization. For the imperfect prototype filter induced interference, the authors in [12] proposed an analytical expression of the overall distortion in non-dispersive channels by considering a finite-length discontinuous prototype filter. However, it has been reported that a well designed prototype filter with moderate length (e.g. overlapping factor $K=4 \sim 6$ ) incurs negligible self-interference ${ }^{1}$ [1], [6].

There have been some investigations in the literature on the dispersive channel induced interference in the form of ICI and/or ISI for FBMC systems. However, most works focused only on simulation-based evaluations and performance comparisons, e.g. [6], [13], [14]. In addition, a few papers claimed that in comparison to OFDM, the FBMC system with well localized prototype filter in time and frequency domain guarantees immunity to dispersive channels [15]; as a result, the low-complexity one-tap frequency domain equalization is applicable to FBMC systems for moderate dispersive channels in the absence of guard time between FBMC symbols [15]. However, there is no theoretical analysis to prove why the circular convolution property holds true in non-CP based FBMC systems as in the CP-OFDM systems. In addition, it is unclear how much performance loss will be anticipated when the circular convolution property is not strictly fulfilled in various channel conditions.

Few works in the literature focused on the advanced channel equalization approaches to reduce the ICI in highly frequency selective channels. Authors in [16] proposed a two stage ordered successive interference cancelation (OSIC) technique. The first stage consists of using the OSIC technique to provide an initial estimation of the transmitted symbols. In the second stage, the rough initial estimation is used to remove ICI and the OSIC technique is then applied again. In addition, the authors in [17] proposed frequency sampling-based (FS) equalizer design techniques for MIMO (multiple-input-multiple-output) FBMC systems. It was shown that significant gain can be achieved at a cost of slightly higher complexity. The authors in [18] have analyzed the effect of multi-tap subcarrier equalization on error performance of precoded MIMO-FBMC systems transmitting through highly frequency selective channel. Their results suggest that subcarrier equalizers with more than three taps do not bring any noticeable improvement in the system performance. In another study, the authors in [19] evaluated the performance of precoding and receiver pro-

\footnotetext{
${ }^{1}$ Indeed, the analytical and simulation results in Section $\mathrm{V}$ show that this type of interference contributes less than to $-40 \mathrm{~dB}$ means square error (MSE) in total.
} 
cessing techniques for multiple access MIMO-FBMC system. Results show that for the uplink transmission, forward error correction is required in addition to receiver processing to obtain satisfactory performance. With channel state and all users information available, the downlink produces better bit error rates comparatively. In addition, both linear and nonlinear transceiver processing approaches for MIMO-FMBC systems were considered in [20] to evaluate the performance of FMBC in terms of bit error rate (BER). It was shown that the linear processing technique cannot offer adequate performance improvement while the non-linear processing can eliminate the error floor effectively.

The aforementioned studies focused on advanced equalization algorithms to eliminate ICI by using simplified/approxmiated models and by partially considering time domain distortion caused by the channel. However, an analytical system model to analyze the impact of doubly dispersive channel is unavailable to date. In addition, it is still an open question as to how much performance penalty will occur in the presence of dispersive channel with specific quantized dispersions (e.g. Doppler spread or delay spread), and in what situation multi-tap channel equalization is required to minimize the ICI/ISI induced performance loss. Furthermore, the blockbased FBMC system (i.e. several consecutive symbols belongs to a block are correlated to each other) is different from the symbol-based OFDM system, the inter-block interference (IBI) is another source of interference which should be taken into consideration in multipath channel environments when no guard interval is available between FBMC transmission blocks.

In this paper, we establish a theoretical framework for FBMC systems by taking into account both frequency and time domain channel dispersions. For our analysis, we first represent the FBMC system model in a vector/matrix form, all types of interference (IBI, ISI and ICI), noise and desired signal estimation bias are derived for two most representative linear channel equalization algorithms: zero-forcing (ZF) and MMSE. We then prove that the circular convolution property between modulated symbols and corresponding channel coefficients in frequency domain can be satisfied in FBMC systems with minor inaccuracies. The MSE of received symbol is derived accordingly. The contributions of this paper are summarized as follows:

- We derive an analytical expression of the FBMC system signal model in a matrix form, in terms of IBI, ISI and ICI, noise and desired signal estimation bias as presented in Section III. MSE due to each term is derived individually in Section IV, and those expressions are given as functions of channel parameters such as Doppler spread and delay spread. Our analysis clearly and quantitatively shows how the channel dispersion degrades system's performance. This analytical framework provides a useful guideline for optimal system design by minimizing the total interference caused by dispersive channels. The work also explains when and why the FBMC system is immune to dispersive channels and how much performance loss will be incurred by a specific channel.

- We provide a mathematical proof to show that the circular convolution relationship between modulated symbols and channel coefficients is a valid assumption for the FBMC system in moderate dispersive channel without CP insertion. This explains why one-tap equalization is sufficient for FBMC in moderate dispersive channels.

- In this paper, we focus our analysis on SISO (singleinput-single-output) system, However, it can be readily extended to MIMO system. In addition, the developed mathematic framework serves as a basis for different types of performance analysis. In the numerical examples, we adopt two sets of the most representative channels: 3GPP (3rd Generation Partnership Project) LTE (long term evolution) channels and IEEE (Institute of Electrical and Electronics Engineers) 802.11 channels. However, the analysis is generic and broadly applicable to different channel models.

Notations: Vectors and matrices are denoted by lowercase and uppercase bold letters, and $\{\cdot\}^{H},\{\cdot\}^{T},\{\cdot\}^{*}$ stand for the Hermitian conjugate, transpose and conjugate operation, respectively. $\mathcal{E}\{\mathbf{A}\}$ denotes the expectation operation of $\mathbf{A}$. We use $\Re\{\mathbf{A}\}$ and $\Im\{\mathbf{A}\}$ to denote taking the real and imaginary part of the scalar/variable/matrix $\mathbf{A}$. $\|\mathbf{A}\|$ refers to the Frobenius norm of matrix A. $\mathbf{I}_{m \times m}$ refers to $m$ dimension identity matrix and for some cases the subscript will be dropped for simplification whenever no ambiguity arises. $\mathbf{1}_{m \times n}$ means an $m \times n$ matrix with all its element being 1. $\operatorname{Tr}\{\mathbf{A}\}$ denotes taking the trace of matrix $\mathbf{A}$. We use * as a linear convolution operation of two vectors/matrices. In addition, We use $\{-\}$ and $\{\sim\}$ over a symbol to refer to the real and imaginary branches related scalars/vectors/matrices, respectively.

\section{BACKGROUND}

\section{A. FBMC/OQAM System}

Without loss of generality, we assume that the transmitting data is modulated to QAM (quadrature amplitude modulation) symbols. To satisfy the orthogonality requirements, the FBMC system has to transmit a real symbol every half symbol duration, resulting in the so-called FBMC/OQAM (offset QAM) system [21]. Alternatively, it could be implemented by shifting the prototype filter while extending the real and imaginary parts of the symbol into the whole symbol duration [15], [22], which is equivalent to the traditional FBMC/OQAM implementation. One advantage of this alternative is that it can avoid the staggered processing of mapping the complex QAM symbols into OQAM symbols [21]. However, the real and imaginary branch should be processed independently, as depicted in Fig. 1. In this paper, we will use this alternative implementation for our analysis. The baseband discrete signal at $i$-th sample of $m$-th FBMC symbol at the output of a synthesis filterbank is expressed by [15]

$$
\begin{aligned}
x_{i}[m] & =\frac{1}{\sqrt{N}} \bar{g}_{i}[m] * \sum_{n=0}^{N} \bar{a}_{m, n} e^{\frac{j \pi(n+2 m)}{2}} e^{\frac{j 2 \pi n i}{N}} \\
& +j \cdot \frac{1}{\sqrt{N}} \bar{g}_{i-N / 2}[m] * \sum_{n=0}^{N} \tilde{a}_{m, n} e^{\frac{j \pi(n+2 m)}{2}} e^{\frac{j 2 \pi n i}{N}}(1)
\end{aligned}
$$


where $m, n$ and $N$ are the time index for the FBMC symbol, the index of subcarrier and the total number of subcarriers in each FBMC symbol, respectively. $\bar{a}_{m, n}$ and $\tilde{a}_{m, n}$ are the real and imaginary part of the input QAM symbol $a_{m, n}$, i.e. $a_{m, n}=\bar{a}_{m, n}+j \tilde{a}_{m, n} \cdot \bar{g}_{i}[m]=\bar{g}[m N+i]$ and $\bar{g}_{i-N / 2}[m]=$ $\bar{g}[m N+i-N / 2]$ are the prototype filters of the real and imaginary branches, respectively, which will be introduced in Section II-C in detail. From Eq. (1) we can see that rather than offsetting the QAM symbols, this model shifts the prototype filter instead.

\section{B. Doubly dispersive Channel Model}

We consider tap-delay-line based channel model that has $L$ taps with its $l$-th tap power being $\rho_{l}^{2}$, which keeps constant during the transmission of the whole FBMC block. Each block contains $M$ FBMC symbols and we assume that the channel is static in one FBMC symbol duration, then the channel for the $m$-th FBMC symbol can be expressed in a vector form as

$$
\begin{aligned}
\mathbf{h}_{m} & =\left[h_{m, 0}, h_{m, 1}, \cdots, h_{m, L-1}\right]^{T} \\
& =\left[\rho_{0} z_{m, 0}, \rho_{1} z_{m, 1}, \cdots, \rho_{L-1} z_{m, L-1}\right]^{T}
\end{aligned}
$$

where $h_{m, l}=\rho_{l} z_{m, l}$ is the $l$-th tap in the time domain channel impulse response, and the complex random variable $z_{m, l}$ with complex Gaussian distribution as $\mathcal{C N}(0,1)$ is a small-scale multipath fading factor of the $l$-th tap of the channel. We assume $z_{m, l 1}$ is independent of $z_{m, l 2}$ for $l 1 \neq l 2$. To show the time domain channel dispersion, the $l$-th tap multipath fading factor at the $i$-th sample of of the $m$-th FBMC symbols can be expressed by [23], [24]:

$$
z_{i, l}=\lambda_{m-i} z_{m, l}+e_{m-i, l}
$$

(3) is also called Jake's model where $\lambda_{m-i}=\mathcal{J}_{0}\left[2 \pi f_{D}(m-\right.$ i) $\Delta T$ ] is the temporal correlation factor [23], [24]. $\mathcal{J}_{0}$ is the zero-order Bessel function of first kind, $f_{D}$ and $\Delta T$ refer to the Doppler spread and the FBMC symbol duration, respectively, and $e_{m-i}$ denotes the channel mismatch vector with each element being modeled as $\mathcal{C N}\left(0,1-\lambda_{m-i}^{2}\right)$ [23], [24]. Note that $f_{D}$ is a parameter to measure the channel dispersion in time domain, larger $f_{D}$ leads to a smaller $\lambda_{m-i}$ and the channel between two consecutive symbols are more uncorrelated.

On the other hand, the channel frequency domain dispersion (i.e. Doppler spread) can be measured by root mean square (RMS) delay spread as $\tau_{R M S}=\sqrt{\sum_{l=0}^{L-1} \rho_{l}^{2} l^{2} / \rho_{\text {tot }}-\tau_{0}^{2}}$, where $\rho_{t o t}=\sum_{l=0}^{L-1} \rho_{l}^{2}$ is the total power of the channel, and $\tau_{0}=\sum_{l=0}^{L-1} \rho_{l}^{2} l / \rho_{t o t}$ is the mean delay [23]. Apparently, larger $\tau_{R M S}$ leads to more frequency selective channels.

\section{Prototype Filters and Filter Matrices}

Let us suppose the overlap factor of the prototype filter is $K$, then the total length of prototype filter $\overline{\mathrm{g}}$ is $K N$, and the filter can be written as

$$
\overline{\mathbf{g}}=\left[\overline{\mathbf{g}}_{0}, \overline{\mathbf{g}}_{1}, \cdots, \overline{\mathbf{g}}_{K-1}\right]=\left[\bar{g}_{0}, \bar{g}_{1}, \cdots, \bar{g}_{K N-1}\right]
$$

with its $k$-th sub-vector $\overline{\mathrm{g}}_{k}$ (for $k=0,1, \cdots, K-1$ ) being defined as

$$
\overline{\mathbf{g}}_{k}=\left[\bar{g}_{k N}, \bar{g}_{k N+1}, \cdots, \bar{g}_{k N+N-1}\right] \in \mathbb{R}^{1 \times N}
$$

The prototype filter will be linearly convolved with the signals in the transmitter and receiver. In order to simplify the derivation, we replace linear convolution operations by matrices multiplications and define a diagonal matrix $\overline{\mathbf{G}}_{k}=$ $\operatorname{diag}\left(\overline{\mathbf{g}}_{k}\right)$ for $k=0,1, \cdots, K-1$. Then the convolution matrix $\overline{\mathbf{C}} \in \mathbb{C}^{(K+M-1) N \times M N}$ becomes

$$
\overline{\mathbf{C}}=\left(\begin{array}{ccccc}
\overline{\mathbf{G}}_{0} & \mathbf{0} & \mathbf{0} & \cdots & \mathbf{0} \\
\overline{\mathbf{G}}_{1} & \overline{\mathbf{G}}_{0} & \mathbf{0} & \cdots & \mathbf{0} \\
\vdots & \overline{\mathbf{G}}_{1} & \overline{\mathbf{G}}_{0} & \cdots & \mathbf{0} \\
\overline{\mathbf{G}}_{K-1} & \vdots & \overline{\mathbf{G}}_{1} & \cdots & \mathbf{0} \\
\overline{\mathbf{0}} & \overline{\mathbf{G}}_{K-1} & \vdots & \cdots & \overline{\mathbf{G}}_{0} \\
\overline{\mathbf{0}} & \mathbf{0} & \overline{\mathbf{G}}_{K-1} & \cdots & \overline{\mathbf{G}}_{1} \\
\vdots & \vdots & \vdots & \ddots & \vdots \\
\overline{\mathbf{0}} & \mathbf{0} & \mathbf{0} & \cdots & \overline{\mathbf{G}}_{K-1}
\end{array}\right)
$$

Note that FBMC system consists of two independent branches as shown by Eq. (1) and Fig. 1, where the imaginary branch prototype filter can be expressed as a shift of the real branch as

$$
\begin{aligned}
& \tilde{\mathbf{g}}=\left[\tilde{\mathbf{g}}_{0}, \tilde{\mathbf{g}}_{1}, \cdots, \tilde{\mathbf{g}}_{K-1}\right]=\left[\tilde{g}_{0}, \tilde{g}_{1}, \cdots, \tilde{g}_{K N-1}\right] \\
& =\left[\bar{g}_{N / 2}, \bar{g}_{N / 2+1}, \cdots, \bar{g}_{K N-1}, \bar{g}_{0}, \bar{g}_{1}, \cdots, \bar{g}_{N / 2-1}\right](7)
\end{aligned}
$$

Following the same method as for the real branch to design $\tilde{\mathbf{G}}_{k}=\operatorname{diag}\left[\tilde{\mathbf{g}}_{k}\right]$ with $\tilde{\mathbf{g}}_{k}=\left[\tilde{g}_{k N}, \tilde{g}_{k N+1}\right.$,

$\left.\cdots, \tilde{g}_{k N+N-1}\right]$. The convolution matrix $\tilde{\mathbf{C}}$ for imaginary branch can be defined with the same structure as $\overline{\mathbf{C}}$ in Eq. (6) with $\overline{\mathbf{G}}_{k}$ replaced by $\tilde{\mathbf{G}}_{k}$.

Let us define the autocorrelation and cross-correlation matrices of $\overline{\mathbf{C}}$ and $\tilde{\mathbf{C}}$ as

$$
\overline{\overline{\mathbf{D}}}=\overline{\mathbf{C}}^{H} \overline{\mathbf{C}}, \quad \tilde{\overline{\mathbf{D}}}=\overline{\mathbf{C}}^{H} \tilde{\mathbf{C}}, \quad \tilde{\tilde{\mathbf{D}}}=\tilde{\mathbf{C}}^{H} \tilde{\mathbf{C}}, \quad \overline{\tilde{\mathbf{D}}}=\tilde{\mathbf{C}}^{H} \overline{\mathbf{C}}
$$

where the matrices $\overline{\overline{\mathbf{D}}}, \tilde{\overline{\mathbf{D}}}, \tilde{\tilde{\mathbf{D}}}$ and $\overline{\tilde{\mathbf{D}}}$ have dimension $M N \times$ $M N$. The four matrices can be split into $M$ row and $M$ column sub-matrices (in total $M^{2}$ sub-matrices) with dimension $N \times N$, respectively, with $\overline{\overline{\mathbf{D}}}_{m, i}, \tilde{\overline{\mathbf{D}}}_{m, i}, \tilde{\tilde{\mathbf{D}}}_{m, i}$ and $\overline{\tilde{\mathbf{D}}}_{m, i}$ being the $m$-th row and $i$-th column sub-matrices.

\section{INTERFERENCE ANALYSIS IN THE PRESENCE OF DOUBLY DISPERSIVE CHANNELS}

The block diagram for both transmitter and receiver of the FBMC system is shown in Fig. 1, where real and imaginary branches are independently and simultaneously processed at both the transmitter and the receiver sides. We will first focus on the real branch derivation, followed by derivations for the imaginary branch.

\section{A. Transmitter Processing}

FBMC takes a block-based processing approach, we assume that each FBMC block contains $M$ FBMC symbols and each symbol has $N$ subcarriers in frequency domain (or 


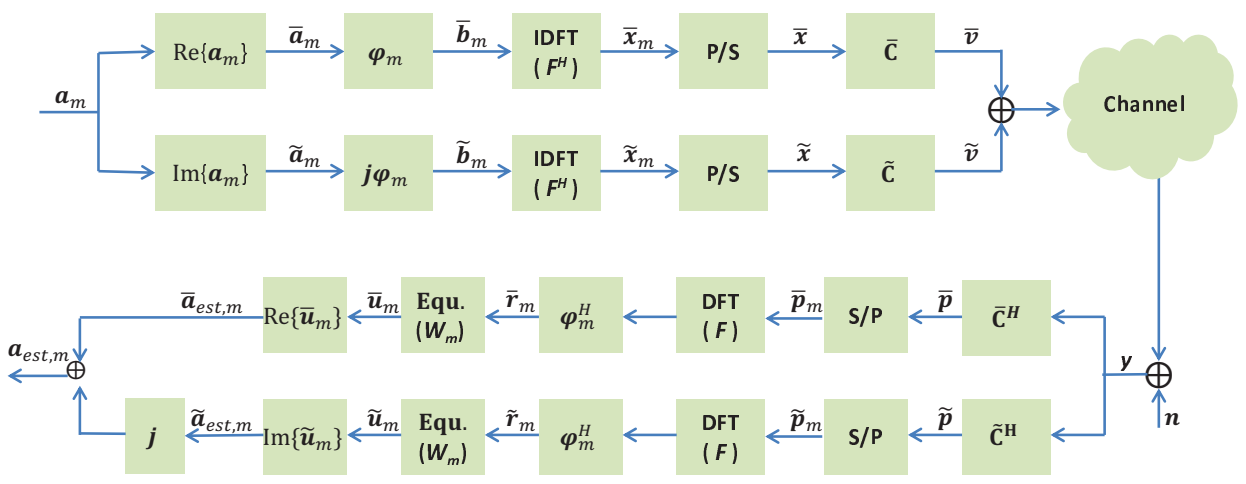

Fig. 1. Blocks diagrams for FBMC transmitter and receiver in matrix operation form.

equivalently $N$ samples in time domain), then in total $M N$ modulated QAM symbols can be transmitted by one FBMC block. We assume the power of modulated signals $a_{m, n}$ (for $m=0,1, \cdots, M-1$ and $n=0,1, \cdots, N-1)$ is $\varrho^{2}$, i.e. $\mathcal{E}\left\{\left\|a_{m, n}\right\|^{2}\right\}=\varrho^{2}$. The modulated symbols $a_{m, n}$ can be expressed in a vector form as $\mathbf{a}_{m}=\overline{\mathbf{a}}_{m}+j \tilde{\mathbf{a}}_{m} \in \mathbb{C}^{N \times 1}$, with $\mathbf{a}_{m}=\left[a_{m, 0}, a_{m, 1}, \cdots, a_{m, N-1}\right]^{T}$ being the $m$-th FBMC symbol. $\overline{\mathbf{a}}_{m}$ and $\tilde{\mathbf{a}}_{m}$ are real and imaginary parts of $\mathbf{a}_{m}$, respectively.

1) Real Branch Phase Shifting: According to Fig. 1, the first step is multiplying $\overline{\mathbf{a}}_{m}$ by phase shifter $\boldsymbol{\varphi}_{m}$ symbol-bysymbol, i.e.,

$$
\overline{\mathbf{b}}_{m}=\boldsymbol{\varphi}_{m} \overline{\mathbf{a}}_{m} \in \mathbb{C}^{N \times 1}, \quad \text { for } \quad m=0,1, \cdots, M-1
$$

where $\varphi_{m}$ is a diagonal matrix with its $n$-th diagonal element being $\varphi_{m, n}=e^{-j \pi(n+2 m) / 2}$, i.e. $\boldsymbol{\varphi}_{m}=$ $\operatorname{diag}\left[e^{-j \pi(0+2 m) / 2}, e^{-j \pi(1+2 m) / 2}, \cdots, e^{-j \pi(N-1+2 m) / 2}\right]$.

2) Real Branch IDFT Processing: The signal after phase shifting will pass to an $N$-size IDFT (inverse discrete Fourier transform) block, then the real branch output after IDFT processing is

$$
\begin{aligned}
\overline{\mathbf{x}} & =\left[\overline{\mathbf{x}}_{0} ; \overline{\mathbf{x}}_{1} ; \cdots ; \overline{\mathbf{x}}_{M-1}\right] \\
& =\left[\mathbf{F}^{H} \overline{\mathbf{b}}_{0} ; \mathbf{F}^{H} \overline{\mathbf{b}}_{1} ; \cdots ; \mathbf{F}^{H} \overline{\mathbf{b}}_{M-1}\right] \in \mathbb{C}^{M N \times 1}
\end{aligned}
$$

where $\overline{\mathbf{x}}_{m}=\mathbf{F}^{H} \overline{\mathbf{b}}_{m} . \mathbf{F}^{H}$ is the normalized $N$-point IDFT matrix with $\mathbf{F}^{H}=\mathbf{F}^{-1}$.

3) Real Branch Prototype Filtering: The output of IDFT $\mathbf{x}_{k}$ is linearly convolved with the prototype filter $\overline{\mathbf{g}}_{m}$, which can be expressed as

$$
\overline{\mathbf{v}}=\overline{\mathbf{C}} \overline{\mathbf{x}} \in \mathbb{C}^{(M+K-1) N \times 1}
$$

where the real branch convolution matrix $\overline{\mathbf{C}}$ is defined in (6). Note that the output of the filter will have $(K-1) N$ more samples than the input due to the linear convolution operation.

4) Imaginary Branch Processing: Following the same derivation as for the real branch in III-A1, III-A2 and III-A3, we obtain the imaginary branch signal as follows:

$$
\tilde{\mathbf{v}}=\tilde{\mathbf{C}} \tilde{\mathbf{x}} \in \mathbb{C}^{(M+K-1) N \times 1}
$$

with $\tilde{\mathbf{C}}$ being defined after Eq. (6) and

$$
\begin{aligned}
\tilde{\mathbf{x}} & =\left[\tilde{\mathbf{x}}_{0} ; \tilde{\mathbf{x}}_{1} ; \cdots ; \tilde{\mathbf{x}}_{M-1}\right] \\
& =\left[\mathbf{F}^{H} \tilde{\mathbf{b}}_{0} ; \mathbf{F}^{H} \tilde{\mathbf{b}}_{1} ; \cdots ; \mathbf{F}^{H} \tilde{\mathbf{b}}_{M-1}\right] \in \mathbb{C}^{M N \times 1}
\end{aligned}
$$

with $\tilde{\mathbf{b}}_{m}=j \varphi_{m} \tilde{\mathbf{a}}_{m}$

\section{B. Passing Through Channel and Interference Analysis}

The real and imaginary branch signals $\overline{\mathbf{v}}$ and $\tilde{\mathbf{v}}$ will be added together (i.e. $\mathbf{v}=\overline{\mathbf{v}}+\tilde{\mathbf{v}}$ ) and send to the receiver via the channel. The received signal can be written as

$$
\mathbf{y}=\mathbf{h} * \mathbf{v}+\mathbf{n}=\mathbf{h} *(\overline{\mathbf{v}}+\tilde{\mathbf{v}})+\mathbf{n}
$$

where $\mathbf{n}$ is the Gaussian noise and its elements have zero mean and variance $\sigma^{2} . \mathbf{h}$ is the time domain channel impulse response in vector form. Using (2), we define the $l$ th tap multipath fading factor of channel in a matrix form as: $\mathbf{Z}_{l}=\operatorname{diag}\left[z_{0, l} \mathbf{1}_{1 \times N}, z_{1, l} \mathbf{1}_{1 \times N}, \cdots, z_{M+K-1, l} \mathbf{1}_{1 \times N}\right] \in$ $\mathbb{C}^{(M+K-1) N \times(M+K-1) N}$. The definition of $\mathbf{Z}_{l}$ implies that the $N$ samples in the $m$-th FBMC symbol experience the same channel (e.g. $\left.z_{m, l}\right)$ and samples in different symbols will pass through different channels (e.g. $z_{i, l}, m \neq i$ ), then we can change (14) to

$$
\mathbf{y}=\sum_{l=0}^{L-1} \rho_{l} \mathbf{Z}_{l}\left(\overline{\mathbf{v}}^{\downarrow l}+\tilde{\mathbf{v}}^{\downarrow l}\right)+\mathbf{v}_{I B I}+\mathbf{n}
$$

where

$$
\mathbf{v}_{I B I}=\sum_{l=0}^{L-1} \rho_{l} \mathbf{Z}_{l} \mathbf{y}_{B, l}
$$

is the IBI caused by channel multipath effect with $\mathbf{y}_{B, l}=$ $\left[\mathbf{y}_{p, l} ; \mathbf{0}_{[(M+K-l) N-l] \times 1]}\right.$ and $\mathbf{y}_{p, l} \in \mathbb{C}^{l \times 1}$ is the interfering signal from the last FBMC block. $\overline{\mathbf{v}}^{\downarrow l}$ and $\tilde{\mathbf{v}}^{\downarrow l}$ are the $l$-sample delay of $\overline{\mathbf{v}}$ and $\tilde{\mathbf{v}}$ with zero padding in the front. They can be expressed as $\overline{\mathbf{v}}^{\downarrow l}=\left[\mathbf{0}_{l \times 1} ; \overline{\mathbf{v}}_{f, l}\right]$ and $\tilde{\mathbf{v}}^{\downarrow l}=\left[\mathbf{0}_{l \times 1} ; \tilde{\mathbf{v}}_{f, l}\right]$, respectively, $\overline{\mathbf{v}}_{f, l}$ and $\tilde{\mathbf{v}}_{f, l}$ denote the first $(M+K-1) N-l$ elements of $\overline{\mathbf{v}}$ and $\tilde{\mathbf{v}}$, respectively.

By using Eqs. (11) and (12), we can write $\overline{\mathbf{v}}^{\downarrow l}=\overline{\mathbf{C}}^{\downarrow l} \overline{\mathbf{x}}$ and $\tilde{\mathbf{v}}^{\downarrow l}=\tilde{\mathbf{C}} \tilde{\mathbf{C}}^{\downarrow l} \tilde{\mathbf{x}}$, where $\overline{\mathbf{C}}^{\downarrow l}=\left[\mathbf{0}_{l \times M N} ; \overline{\mathbf{C}}_{f, l}\right]$ and $\tilde{\mathbf{C}}^{\downarrow l}=$ $\left[\mathbf{0}_{l \times M N} ; \tilde{\mathbf{C}}_{f, l}\right]$ with $\overline{\mathbf{C}}_{f, l}$ and $\tilde{\mathbf{C}}_{f, l}$ are the first $(M+K-$ 1) $N-l$ rows of $\overline{\mathbf{C}}$ and $\tilde{\mathbf{C}}$, respectively. Then we can rewritten (15) as

$$
\mathbf{y}=\sum_{l=0}^{L-1} \rho_{l} \mathbf{Z}_{l}\left(\overline{\mathbf{C}}^{\downarrow l} \overline{\mathbf{x}}+\tilde{\mathbf{C}}^{\downarrow l} \tilde{\mathbf{x}}\right)+\mathbf{v}_{I B I}+\mathbf{n}
$$


(17) indicates that as a result of channel multipath effect, the original $\overline{\mathbf{C}}$ and $\tilde{\mathbf{C}}$ are replaced by distorted filters $\overline{\mathbf{C}}^{\downarrow l}$ and $\tilde{\mathbf{C}}^{\downarrow l}$, respectively. In order to demonstrate the relationship of the distortion and the multipath effect on the FBMC system, we first introduce a block diagonal exchanging matrix $\mathbf{E}_{l} \in$ $\mathbb{R}^{M N \times M N}$ as follows

$$
\mathbf{E}_{l}=\left(\begin{array}{cccc}
\mathbf{E}_{s u b, l} & \mathbf{0} & \cdots & \mathbf{0} \\
\mathbf{0} & \mathbf{E}_{s u b, l} & \cdots & \mathbf{0} \\
\vdots & \vdots & \ddots & \vdots \\
\mathbf{0} & \mathbf{0} & \cdots & \mathbf{E}_{s u b, l}
\end{array}\right)
$$

with $\mathbf{E}_{s u b, l}=\left[\mathbf{0}_{l \times(N-l)}, \mathbf{I}_{l \times l} ; \mathbf{I}_{(N-l) \times(N-l)}, \mathbf{0}_{(N-l) \times l}\right] \in$ $\mathbb{R}^{N \times N}$. By using $\mathbf{E}_{l}^{T} \mathbf{E}_{l}=\mathbf{I}$, we have

$$
\overline{\mathbf{v}}^{\downarrow l}=\overline{\mathbf{C}}^{\downarrow l} \mathbf{E}_{l}^{T} \mathbf{E}_{l} \overline{\mathbf{x}}=\overline{\mathbf{C}}_{e}^{\downarrow l} \overline{\mathbf{x}}_{e}^{\downarrow l}
$$

where $\overline{\mathbf{C}}_{e}^{\downarrow l}=\overline{\mathbf{C}}^{\downarrow l} \mathbf{E}_{l}^{T}$ and $\overline{\mathbf{x}}_{e}^{\downarrow l}=\mathbf{E}_{l} \overline{\mathbf{x}}$. The functions $\mathbf{E}_{l}^{T}$ and $\mathbf{E}_{l}$ are used to exchange the locations of elements of $\overline{\mathbf{C}}^{\downarrow l}$ and $\overline{\mathbf{x}}$. Specifically, for $\overline{\mathbf{x}}$, by multiplying the permutation matrix $\mathbf{E}_{l}$, the last $l$ symbols of its each sub-vector $\overline{\mathbf{x}}_{m}$ will be moved to the front, i.e.

$$
\overline{\mathbf{x}}_{e, m}^{\downarrow l}=\left[x_{m, N-l} \cdots, x_{m, N-1}, x_{m, 0}, \cdots, x_{m, N-l-1}\right]^{T}
$$

and

$$
\overline{\mathbf{x}}_{e}^{\downarrow l}=\left[\overline{\mathbf{x}}_{e, 0}^{\downarrow l} ; \overline{\mathbf{x}}_{e, 1}^{\downarrow l} ; \cdots ; \overline{\mathbf{x}}_{e, M-1}^{\downarrow l}\right]
$$

The effect of multiplying $\mathbf{E}_{l}^{T}$ with $\overline{\mathbf{C}}^{\downarrow l}$ is similar. $\mathbf{E}_{l}^{T}$ only changes the elements locations in $\overline{\mathbf{C}}^{\downarrow l}$. All the non-zero elements in $\overline{\mathbf{C}}_{e}^{\downarrow l}$ are comprised of the elements of prototype filter $\overline{\mathrm{g}}$ (i.e. $\bar{g}_{i}, i=0,1, \cdots, K N-1$ ), which is the same as matrix $\overline{\mathbf{C}}$. Specifically, the counterpart elements in $\overline{\mathbf{C}}_{e}^{\downarrow l}$ are delayed by $l$ elements in $\overline{\mathrm{g}}$ comparing with $\overline{\mathbf{C}}$, e.g., if the non-zero $i$-th row and $k$-th column element of $\overline{\mathbf{C}}$ is $\bar{g}_{n}$, then the element of $\overline{\mathbf{C}}_{e}^{\Downarrow l}$ at the same location will be $\bar{g}_{n+l}$. The difference of $\bar{g}_{n}$ and $\bar{g}_{n+l}$ is very small with $N \gg L$ since the values of adjacent elements of the prototype filter are close to each other.

Similarly, for $\tilde{\mathbf{v}}^{\downarrow l}$ we have

$$
\tilde{\mathbf{v}}^{\downarrow l}=\tilde{\mathbf{C}}^{\downarrow l} \mathbf{E}_{l}^{T} \mathbf{E}_{l} \tilde{\mathbf{x}}=\tilde{\mathbf{C}}_{e}^{\downarrow l} \tilde{\mathbf{x}}_{e}^{\downarrow l}
$$

where $\tilde{\mathbf{C}}_{e}^{\downarrow l}$ and $\tilde{\mathbf{x}}_{e}^{\downarrow l}$ have exactly the same structure as $\overline{\mathbf{C}}_{e}^{\downarrow l}$ and $\overline{\mathbf{x}}_{e}^{\Downarrow l}$, except replacing all upper-scripts $\{\dot{*}\}$ by $\{\tilde{\}}$. Substituting (19) and (22) into (17), we have

$$
\mathbf{y}=\sum_{l=0}^{L-1} \rho_{l} \mathbf{Z}_{l}\left(\overline{\mathbf{C}}_{e}^{\downarrow l} \overline{\mathbf{x}}_{e}^{\downarrow l}+\tilde{\mathbf{C}}_{e}^{\downarrow l} \tilde{\mathbf{x}}_{e}^{\downarrow l}\right)+\mathbf{v}_{I B I}+\mathbf{n}
$$

As discussed earlier, non-zero elements of $\overline{\mathbf{C}}_{e}^{\downarrow l}$ and $\overline{\mathbf{C}}$ are very close. In order to show the error caused by the multipath on the filter distortion, we define

$$
\overline{\mathbf{C}}_{e}^{\downarrow l}=\overline{\mathbf{C}}+\Delta \overline{\mathbf{C}}^{\downarrow l}, \quad \tilde{\mathbf{C}}_{e}^{\downarrow l}=\tilde{\mathbf{C}}+\Delta \tilde{\mathbf{C}}^{\downarrow l}
$$

The diagonal matrix $\mathbf{Z}_{l}$ in (23), which is caused by the channel fading, boils down to a unitary matrix and can thus be omitted if the channel is static during the whole FBMC block transmission, i.e., there is no performance loss caused by channel frequency dispersion on FBMC system. However, in high mobility environments, $\mathbf{Z}_{l}$ is a non-unitary diagonal matrix and its impact is not negligible. Let us model $\mathbf{Z}_{l}$ as:

$$
\mathbf{Z}_{l}=z_{m, l} \mathbf{I}+\Delta \mathbf{Z}_{l}
$$

with $\Delta \mathbf{Z}_{l}=\Delta \mathbf{Z}_{1, l}+\Delta \mathbf{Z}_{2, l}$, where $\Delta \mathbf{Z}_{1, l}=z_{m, l} \operatorname{diag}[(1-$ $\left.\lambda_{m}\right) \mathbf{1}_{1 \times N},\left(1-\lambda_{m-1}\right) \mathbf{1}_{1 \times N}, \cdots,\left(1-\lambda_{1}\right) \mathbf{1}_{1 \times N}, \mathbf{0}_{1 \times N},(1-$ $\left.\left.\lambda_{1}\right) \mathbf{1}_{1 \times N}, \cdots,\left(1-\lambda_{M+K-1-m}\right) \mathbf{1}_{1 \times N}\right]$ and $\Delta \mathbf{Z}_{2, l}=$ $\operatorname{diag}\left[e_{m, l} \mathbf{1}_{1 \times N}, e_{m-1, l} \mathbf{1}_{1 \times N}\right.$,

$\left.\cdots, e_{1, l} \mathbf{1}_{1 \times N}, \mathbf{0}_{1 \times N}, e_{1, l} \mathbf{1}_{1 \times N}, \cdots, e_{M+K-1-m, l} \cdot \mathbf{1}_{1 \times N}\right]$. With a small Doppler spread $f_{D}$, the variance of each element of $\Delta \mathbf{Z}_{1, l}$ is always much smaller than $\Delta \mathbf{Z}_{2, l}$, i.e. $\left(1-\lambda_{l}\right)^{2}<<$ $1-\lambda_{l}^{2}$ when $\lambda_{l}$ is close to one.

Substituting (24) and (25) into (23) leads to

$$
\mathbf{y}=\sum_{l=0}^{L-1} \rho_{l} z_{m, l}\left(\overline{\mathbf{C}} \overline{\mathbf{x}}_{e}^{\downarrow l}+\tilde{\mathbf{C}}_{e}^{\downarrow l}\right)+\mathbf{v}_{I B I}+\mathbf{v}_{f m}+\mathbf{v}_{f d}+\mathbf{n}
$$

where the filter mismatch error $\mathbf{v}_{f m}$ due to the channel multipath effect (time domain dispersion) can be written as

$$
\mathbf{v}_{f m}=\sum_{l=0}^{L-1} \rho_{l} \mathbf{Z}_{m, l}\left(\Delta \overline{\mathbf{C}}^{\downarrow l} \overline{\mathbf{x}}_{e}^{\downarrow l}+\Delta \tilde{\mathbf{C}}^{\downarrow l} \tilde{\mathbf{x}}_{e}^{\downarrow l}\right)
$$

and the channel fading (frequency domain dispersion) caused error $\mathbf{v}_{f d}$ is defined as

$$
\mathbf{v}_{f d}=\sum_{l=0}^{L-1} \rho_{l} \Delta \mathbf{Z}_{l}\left(\overline{\mathbf{C}}_{e}^{\downarrow l} \overline{\mathbf{x}}_{e}^{\downarrow l}+\tilde{\mathbf{C}}_{e}^{\downarrow l} \tilde{\mathbf{x}}_{e}^{\downarrow l}\right)
$$

Note that the cross error term $\sum_{l=0}^{L-1} \rho_{l} \Delta \mathbf{Z}_{l}\left(\Delta \overline{\mathbf{C}}^{\downarrow l} \overline{\mathbf{x}}_{e}^{\downarrow l}+\right.$ $\left.\Delta \tilde{\mathbf{C}}^{\downarrow l} \tilde{\mathbf{x}}_{e}^{\downarrow l}\right)$ in Eq. (26) is omitted since it is a second order error and negligible comparing with the error terms $\mathbf{v}_{f m}$ and $\mathbf{v}_{f d}$. Apparently, the value of $\mathbf{v}_{f m}$ depends on the delay spread of the channel and a larger $\tau_{R M S}$ results in a stronger interference to the system. On the other hand, $\mathbf{v}_{f d}$ depends on the Doppler spread of the system and a larger $f_{D}$ leads to a larger $\mathbf{v}_{f d}$.

\section{Receiver processing}

1) Receiver Filtering of Real Branch: Passing the received signal $\mathbf{y}$ to the prototype filter leads to the following output

$$
\begin{aligned}
\overline{\mathbf{p}} & =\overline{\mathbf{C}}^{H} \mathbf{y}=\overline{\mathbf{C}}^{H} \overline{\mathbf{C}} \sum_{l=0}^{L-1} \rho_{l} z_{m, l} \overline{\mathbf{x}}_{e}^{\downarrow l}+\overline{\mathbf{C}}^{H} \tilde{\mathbf{C}} \sum_{l=0}^{L-1} \rho_{l} z_{m, l} \tilde{\mathbf{x}}_{e}^{\downarrow l} \\
& +\overline{\mathbf{C}}^{H}\left(\mathbf{v}_{I B I}+\mathbf{v}_{f d}+\mathbf{v}_{f m}+\mathbf{n}\right)
\end{aligned}
$$

2) DFT Processing and Phase Shifting of Real Branch: Vector $\overline{\mathbf{p}}=\left[\bar{p}_{0}, \bar{p}_{1}, \cdots, \bar{p}_{M N-1}\right]^{T}$ is split into $M$ segments, each of which has $N$ elements to perform the $N$-point DFT and phase shifting operation. Define the $m$-th segment of $\overline{\mathbf{p}}$ as $\overline{\mathbf{p}}_{m}=\left[\bar{p}_{m N}, \bar{p}_{m N+1}, \cdots, \bar{p}_{m N+N-1}\right]^{T}$. We can have the signal before channel equalization as

$$
\overline{\mathbf{r}}_{m}=\boldsymbol{\varphi}_{m}^{H} \mathbf{F} \overline{\mathbf{p}}_{m}
$$


3) Channel Equalization of Real Branch: We assume that one-tap channel equalizer diagonal matrix $\mathbf{W}_{m}$ is applied to the real branch data $\overline{\mathbf{r}}_{m}$ as follows

$$
\overline{\mathbf{u}}_{m}=\mathbf{W}_{m} \varphi_{m}^{H} \mathbf{F} \overline{\mathbf{p}}_{m}
$$

By using (8) and the definition of $\overline{\mathbf{p}}_{m}$, we can expand (31) as sum of desired signals, interference and noise

$$
\begin{aligned}
& \overline{\mathbf{u}}_{m}=\underbrace{\mathbf{W}_{m} \boldsymbol{\varphi}_{m}^{H} \mathbf{F} \sum_{i=0}^{M-1} \overline{\overline{\mathbf{D}}}_{m, i} \sum_{l=0}^{L-1} \rho_{l} z_{m, l} \overline{\mathbf{x}}_{e, i}^{\downarrow l}}_{=\overline{\mathbf{u}}_{R, m}} \\
&+\underbrace{\mathbf{W}_{m} \boldsymbol{\varphi}_{m}^{H} \mathbf{F} \sum_{i=0}^{M-1} \tilde{\overline{\mathbf{D}}}_{m, i} \sum_{l=0}^{L-1} \rho_{l} z_{m, l} \tilde{\mathbf{x}}_{e, i}^{\downarrow l}}_{=\overline{\mathbf{u}}_{I, m}} \\
&+ \underbrace{\mathbf{W}_{m} \boldsymbol{\varphi}_{m}^{H} \mathbf{F} \overline{\mathbf{C}}_{m}^{H} \mathbf{v}_{I B I}}_{=\overline{\mathbf{u}}_{I B I, m}}+\underbrace{\mathbf{W}_{m} \boldsymbol{\varphi}_{m}^{H} \mathbf{F} \overline{\mathbf{C}}_{m}^{H} \mathbf{v}_{f d}}_{=\overline{\mathbf{u}}_{f d, m}} \\
&+\underbrace{\mathbf{W}_{m} \boldsymbol{\varphi}_{m}^{H} \mathbf{F} \overline{\mathbf{C}}_{m}^{H} \mathbf{v}_{f m}}_{=\overline{\mathbf{u}}_{f m, m}}+\underbrace{\mathbf{W}_{m} \boldsymbol{\varphi}_{m}^{H} \mathbf{F} \overline{\mathbf{C}}_{m}^{H} \mathbf{n}}_{=\overline{\mathbf{u}}_{m o i s e, m}}
\end{aligned}
$$

where $\overline{\overline{\mathbf{D}}}_{m, i}$ and $\tilde{\overline{\mathbf{D}}}_{m, i}$ are the $m$-th row $i$-th column submatrices of $\overline{\bar{D}}$ and $\tilde{\bar{D}}$, respectively, as defined after Eq. (8). Eq. (32) includes 6 terms, the third term $\overline{\mathbf{u}}_{I B I, m}$, the fourth term $\overline{\mathbf{u}}_{f d, m}$ and fifth terms $\overline{\mathbf{u}}_{f m, m}$ are interference caused by the doubly dispersive channel. The last term $\overline{\mathbf{u}}_{\text {noise } m}$ is the noise that has been processed by prototype filter, DFT and phase shifter. The second term $\overline{\mathbf{u}}_{I, m}$ is the interference generated by the imaginary part of signals (i.e. $\tilde{\mathbf{a}}_{m, n}$ ). The first term $\overline{\mathbf{u}}_{R, m}$ is the only term that contains the desired symbols (i.e. $\overline{\mathbf{a}}_{m, n}$ ).

We will show that channel circular convolution property holds for $\overline{\mathbf{u}}_{R, m}$ and $\overline{\mathbf{u}}_{I, m}$. Since the derivation on $\overline{\mathbf{u}}_{R, m}$ and $\overline{\mathbf{u}}_{I, m}$ are similar, we will only give $\overline{\mathbf{u}}_{R, m}$ derivation in detail.

We prove that the channel coefficients and the transmitted signal $\overline{\mathbf{a}}_{i}$ for $i=0,1, \cdots, M-1$ in $\overline{\mathbf{u}}_{R, m}$ satisfies the circular convolution property and therefore can be written as the following point-wise multiplication form in the frequency domain

$$
\overline{\mathbf{u}}_{R, m}=\sum_{i=0}^{M-1} \mathbf{W}_{m} \overline{\overline{\mathbf{Q}}}_{m, i} \mathbf{H}_{m} \overline{\mathbf{a}}_{i}
$$

with $\overline{\mathbf{Q}}_{m, i}=\boldsymbol{\varphi}_{m}^{H} \mathbf{F} \overline{\bar{D}}_{m, i} \mathbf{F}^{H} \boldsymbol{\varphi}_{i} \cdot \mathbf{H}_{m}=\sqrt{N} \operatorname{diag}\left(\mathbf{F} \mathbf{h}_{m}\right)$ is the frequency domain channel coefficients in diagonal matrix form.

Proof: See Appendix A.

Clearly, with diagonal matrix $\mathbf{H}_{m}$, the multiplication $\mathbf{H}_{m} \overline{\mathbf{a}}_{i}$ implies that the channel coefficients and symbols in the corresponding subcarriers perform point-wise multiplications. However, even though this circular convolution property holds, we will show that one-tap channel equalization will bring ICI due to the filter operation (i.e., non-diagonal matrix $\overline{\overline{\mathbf{Q}}}_{m, i}$ ) between equalizer $\mathbf{W}_{m}$ and the channel $\mathbf{H}_{m}$.

According to the orthogonality of FBMC, with infinite filter length, e.g. $K \rightarrow \infty, \overline{\overline{\mathbf{Q}}}_{m, i}$ have the following properties:

$$
\overline{\overline{\mathbf{Q}}}_{m, i}=\left\{\begin{array}{l}
\mathbf{I}+j \Im\left\{\overline{\overline{\mathbf{Q}}}_{m, m}\right\} \quad \text { for } i=m \\
j \Im\left\{\overline{\overline{\mathbf{Q}}}_{m, i}\right\} \text { for } i \neq m
\end{array}\right.
$$

where $\Im\{\cdot\}$ means taking the imaginary part operation. Eq. (34) implies that the real part of $\overline{\mathbf{Q}}_{m, m}$ is an identity matrix for $i=m$; while for $i \neq m, \overline{\mathbf{Q}}_{m, i}$ is an imaginary matrix. Substituting (34) into (33) yields

$$
\overline{\mathbf{u}}_{R, m}=\mathbf{W}_{m} \mathbf{H}_{m} \overline{\mathbf{a}}_{m}+j \sum_{i=0}^{M-1} \mathbf{W}_{m} \Im\left(\overline{\overline{\mathbf{Q}}}_{m, i}\right) \mathbf{H}_{m} \overline{\mathbf{a}}_{i}
$$

which shows that the desired signal $\overline{\mathbf{a}}_{m}$ has been successfully extracted.

As shown in the first term of equation (35) (i.e., $\mathbf{W}_{m} \mathbf{H}_{m} \overline{\mathbf{a}}_{m}$ ), the channel frequency response (diagonal matrix $\left.\mathbf{H}_{m}\right)$ and the transmitted signal $\left(\overline{\mathbf{a}}_{m}\right)$ has been written as a point-wise multiplication. This implies that the circular convolution property holds for FBMC system in the real domain only as there is an additive second term (i.e., intrinsic interference) $j \sum_{i=0}^{M-1} \mathbf{W}_{m} \Im\left(\overline{\overline{\mathbf{Q}}}_{m, i}\right) \mathbf{H}_{m} \overline{\mathbf{a}}_{i}$ in equation (35). However, with a complex-valued channel $\mathbf{H}_{m}, j \sum_{i=0}^{M-1} \mathbf{W}_{m} \Im\left(\overline{\overline{\mathbf{Q}}}_{m, i}\right) \mathbf{H}_{m} \overline{\mathbf{a}}_{i}$ is a complex-valued matrix since $\Im\left(\overline{\mathbf{Q}}_{m, i}\right)$ is not a diagonal matrix. Taking the real part on $\overline{\mathbf{u}}_{R, m}$ in the following stage cannot totally eliminate the interference, i.e., the ICI exists in the system even with circular convolution property holding.

Next, we consider two most widely used channel equalizers: $\mathrm{ZF}$ and MMSE equalizer [25], [26]

$$
\mathbf{W}_{m}=\mathbf{H}_{m}^{H}\left(\mathbf{H}_{m} \mathbf{H}_{m}^{H}+\nu \sigma^{2} / \varrho^{2} \mathbf{I}\right)^{-1}
$$

where $\nu$ is a parameter defined by

$$
\nu=\left\{\begin{array}{cc}
0 & \mathrm{ZF} \text { receiver } \\
1 & \mathrm{MMSE} \text { receiver }
\end{array}\right.
$$

Note that $\mathbf{W}_{m}$ is a diagonal matrix for either ZF or MMSE equalizer. Now let us consider the $n$-th element of $\overline{\mathbf{u}}_{R, m}$ in (35)

$$
\bar{u}_{R, m, n}=\beta_{m, n} \bar{a}_{m, n}+j W_{m, n} \sum_{i=0}^{M-1} \Im\left\{\overline{\overline{\mathbf{q}}}_{m, i, n}\right\} \mathbf{H}_{m} \overline{\mathbf{a}}_{i}
$$

with

$$
\beta_{m, n}=W_{m, n} H_{m, n}=\frac{\left|H_{m, n}\right|^{2}}{\left|H_{m, n}\right|^{2}+\nu \sigma^{2} / \varrho^{2}}
$$

and $\overline{\overline{\mathbf{q}}}_{m, i, n}$ is the $n$-th row of matrix $\overline{\mathbf{Q}}_{m, i} . W_{m, n}$ and $H_{m, n}$ are the $n$-th diagonal element of $\mathbf{W}_{m}$ and $\mathbf{H}_{m}$, respectively. In order to show the channel frequency selectivity caused interference, let us define the differences of the channel coefficients in the $n$-th subcarrier with the other subcarriers in the following matrix form

$$
\Delta \mathbf{H}_{m, n}=\mathbf{H}_{m}-H_{m, n} \mathbf{I}
$$

then we have $W_{m, n} \mathbf{H}_{m}=W_{m, n} \Delta \mathbf{H}_{m, n}+\beta_{m, n} \mathbf{I}$, substituting it into (38) results in

$$
\begin{aligned}
\bar{u}_{R, m, n} & =\beta_{m, n} \bar{a}_{m, n}+\bar{u}_{R, i n t r i, m, n} \\
& +j W_{m, n} \sum_{i=0}^{M-1} \Im\left\{\overline{\mathbf{q}}_{m, i, n}\right\} \Delta \mathbf{H}_{m, n} \overline{\mathbf{a}}_{i}
\end{aligned}
$$

where $\bar{u}_{R, i n t r i, m, n}=j \beta_{m, n} \sum_{i=0}^{M-1} \Im\left\{\overline{\overline{\mathbf{q}}}_{m, i, n}\right\} \overline{\mathbf{a}}_{i}$ is purely imaginary since $\beta_{m, n}, \quad \Im\left\{\overline{\overline{\mathbf{q}}}_{m, i, n}\right\}$ and $\overline{\mathbf{a}}_{i}$ are realvalued. Writing $\bar{u}_{R, i n t r i, m, n}$ as a vector: $\overline{\mathbf{u}}_{R, \text { intri, } m}=$ 
$\left[\bar{u}_{R, \text { intri,m,0 }}, \cdots, \bar{u}_{R, \text { intri,m,N-1 }}\right]^{T} \in \mathbb{I}^{N \times 1}$. Let us further define $\bar{u}_{R, t d, m, n}=j W_{m, n} \sum_{i=0}^{M-1} \Im\left\{\overline{\overline{\mathbf{q}}}_{m, i, n}\right\} \Delta \mathbf{H}_{m, n} \overline{\mathbf{a}}_{i}$, in a vector form as

$$
\overline{\mathbf{u}}_{R, t d, m}=\left[\bar{u}_{R, t d, m, 0}, \cdots, \bar{u}_{R, t d, m, N-1}\right]^{T} \in \mathbb{I}^{N \times 1}
$$

Substituting $\overline{\mathbf{u}}_{R, t d, m}$ and $\overline{\mathbf{u}}_{R, i n t r i, m}$ into (41) and write it as a vector form, yields

$$
\overline{\mathbf{u}}_{R, m}=\boldsymbol{\beta}_{m} \overline{\mathbf{a}}_{m}+\overline{\mathbf{u}}_{R, i n t r i, m}+\overline{\mathbf{u}}_{R, t d, m}
$$

where $\boldsymbol{\beta}_{m}=\operatorname{diag}\left[\beta_{m, 0}, \beta_{m, 1}, \cdots, \beta_{m, N-1}\right]$.

Similarly, the second term $\overline{\mathbf{u}}_{I, m}$ in Eq. (32) can be obtained by following the same derivation and using the same circular convolution property as

$$
\overline{\mathbf{u}}_{I, m}=\overline{\mathbf{u}}_{I, \text { intri,m }}+\overline{\mathbf{u}}_{I, t d, m}
$$

where $\overline{\mathbf{u}}_{I, \text { intri,m }}=\left[\bar{u}_{I, \text { intri,m,0 }}, \cdots, \bar{u}_{I, \text { intri,m,N-1}}\right]^{T}$, with $\bar{u}_{I, i n t r i, m, n}=\beta_{m, n} \sum_{i=0}^{M-1} \tilde{\overline{\mathbf{q}}}_{m, i, n} \tilde{\mathbf{a}}_{i}$, and

$$
\overline{\mathbf{u}}_{I, t d, m}=\left[\bar{u}_{I, t d, m, 0}, \cdots, \bar{u}_{I, t d, m, N-1}\right]^{T} \in \mathbb{I}^{N \times 1}
$$

with its $n$-th element being $\bar{u}_{I, t d, m, n}=$ $W_{m, n} \sum_{i=0}^{M-1} \tilde{\overline{\mathbf{q}}}_{m, i, n} \Delta \mathbf{H}_{m, n} \tilde{\mathbf{a}}_{i}, \quad$ and $\quad \tilde{\overline{\mathbf{q}}}_{m, i, n}$ is the $n$-th row of matrix $\tilde{\overline{\mathbf{Q}}}_{m, i}$ defined as

$$
\tilde{\overline{\mathbf{Q}}}_{m, i}=j \boldsymbol{\varphi}_{m}^{H} \mathbf{F} \tilde{\overline{\mathbf{D}}}_{m, i} \mathbf{F}^{H} \boldsymbol{\varphi}_{i} \in \mathbb{I}^{N \times N}
$$

So far we have calculated both $\overline{\mathbf{u}}_{R, t d, m}$ and $\overline{\mathbf{u}}_{I, t d, m}$ in Eqs. (43) and (44), substituting them into (32) leads to the output of real branch

$$
\begin{aligned}
\overline{\mathbf{u}}_{m} & =\boldsymbol{\beta}_{m} \overline{\mathbf{a}}_{m}+\left(\overline{\mathbf{u}}_{R, \text { intri,m }}+\overline{\mathbf{u}}_{I, \text { intri }, m}\right)+\overline{\mathbf{u}}_{I B I, m} \\
& +\overline{\mathbf{u}}_{t d, m}+\overline{\mathbf{u}}_{f d, m}+\overline{\mathbf{u}}_{f m, m}+\overline{\mathbf{u}}_{\text {noise }, m}
\end{aligned}
$$

where we have defined a new vector

$$
\overline{\mathbf{u}}_{t d, m}=\left(\overline{\mathbf{u}}_{R, t d, m}+\overline{\mathbf{u}}_{I, t d, m}\right)
$$

and $\left(\overline{\mathbf{u}}_{R, \text { intri,m }}+\overline{\mathbf{u}}_{I, \text { intri,m }}\right)$ is the so-called intrinsic interference.

Comparing (47) with (32), except the already defined interference terms $\overline{\mathbf{u}}_{f m, m}, \overline{\mathbf{u}}_{f d, m}$ and $\overline{\mathbf{u}}_{n o i s e, m}$, the first and second terms are written as a sum of desired signal $\boldsymbol{\beta}_{m} \overline{\mathbf{a}}_{m}$, intrinsic interference $\left(\overline{\mathbf{u}}_{R, \text { intri,m }}+\overline{\mathbf{u}}_{I, \text { intri,m }}\right)$ and the extra ICI/ISI $\overline{\mathbf{u}}_{t d, m}$ caused by channel frequency selectivity.

4) Real Branch Symbol Recovery: The intrinsic interference can be removed by taking the real part of $\overline{\mathbf{u}}_{m}$, the estimated real-valued branch of the $m$-th FBMC symbol is

$$
\begin{aligned}
\overline{\mathbf{a}}_{e s t, m} & =\Re\left\{\overline{\mathbf{u}}_{m}\right\}=\boldsymbol{\beta}_{m} \overline{\mathbf{a}}_{m}+\Re\left\{\overline{\mathbf{u}}_{I B I, m}\right\}+\Re\left\{\overline{\mathbf{u}}_{f d, m}\right\} \\
& +\Re\left\{\overline{\mathbf{u}}_{t d, m}\right\}+\Re\left\{\overline{\mathbf{u}}_{f m, m}\right\}+\Re\left\{\overline{\mathbf{u}}_{\text {noise }, m}\right\}
\end{aligned}
$$

5) Receiver Operation for Imaginary Branch: The imaginary branch follows the same procedure and the derivation results are given by:

$$
\begin{aligned}
\tilde{\mathbf{a}}_{e s t, m} & =\Im\left\{\tilde{\mathbf{u}}_{m}\right\}=\boldsymbol{\beta}_{m} \tilde{\mathbf{a}}_{m}+\Im\left\{\tilde{\mathbf{u}}_{I B I, m}\right\}+\Im\left\{\tilde{\mathbf{u}}_{t d, m}\right\} \\
& +\Im\left\{\tilde{\mathbf{u}}_{f d, m}\right\}+\Im\left\{\tilde{\mathbf{u}}_{f m, m}\right\}+\Im\left\{\tilde{\mathbf{u}}_{\text {noise }, m}\right\}
\end{aligned}
$$

where

$$
\begin{aligned}
& \tilde{\mathbf{u}}_{I B I, m}=\mathbf{W}_{m} \boldsymbol{\varphi}_{m}^{H} \mathbf{F} \tilde{\mathbf{C}}_{m}^{H} \mathbf{v}_{I B I}, \quad \tilde{\mathbf{u}}_{t d, m}=\tilde{\mathbf{u}}_{I, t d, m}+\tilde{\mathbf{u}}_{R, t d, m} \\
& \tilde{\mathbf{u}}_{f d, m}=\mathbf{W}_{m} \boldsymbol{\varphi}_{m}^{H} \mathbf{F} \tilde{\mathbf{C}}_{m}^{H} \mathbf{v}_{f d}, \quad \tilde{\mathbf{u}}_{f m, m}=\mathbf{W}_{m} \boldsymbol{\varphi}_{m}^{H} \mathbf{F} \tilde{\mathbf{C}}_{m}^{H} \mathbf{v}_{f m} \\
& \tilde{\mathbf{u}}_{n o i s e, m}=\mathbf{W}_{m} \boldsymbol{\varphi}_{m}^{H} \mathbf{F} \tilde{\mathbf{C}}_{m}^{H} \mathbf{n}
\end{aligned}
$$

$\tilde{\mathbf{u}}_{I, t d, m}$ and $\tilde{\mathbf{u}}_{R, t d, m}$ can be expressed as

$$
\begin{aligned}
& \tilde{\mathbf{u}}_{R, t d, m}=\left[\tilde{u}_{R, t d, m, 0}, \cdots, \tilde{u}_{R, t d, m, N-1}\right]^{T} \in \mathbb{I}^{N \times 1} \\
& \tilde{\mathbf{u}}_{I, t d, m}=\left[\tilde{u}_{I, t d, m, 0}, \cdots, \tilde{u}_{I, t d, m, N-1}\right]^{T} \in \mathbb{I}^{N \times 1}
\end{aligned}
$$

with $\quad \tilde{u}_{R, t d, m, n}=W_{m, n} \sum_{i=0}^{M-1} \Re\left\{\tilde{\tilde{\mathbf{q}}}_{m, i, n}\right\} \Delta \mathbf{H}_{m, n} \tilde{\mathbf{a}}_{i}$ and $\tilde{u}_{I, t d, m, n}=W_{m, n} \sum_{i=0}^{M-1} \overline{\tilde{\mathbf{q}}}_{m, i, n} \Delta \mathbf{H}_{m, n} \overline{\mathbf{a}}_{i}$, where $\tilde{\tilde{\mathbf{q}}}_{m, i, n}$ and $\overline{\tilde{\mathbf{q}}}_{m, i, n}$ are the $n$-th row of matrix $\tilde{\tilde{\mathbf{Q}}}_{m, i}$ and $\overline{\tilde{\mathbf{Q}}}_{m, i}$, respectively, which hold the following properties

$\tilde{\tilde{\mathbf{Q}}}_{m, i}=j \boldsymbol{\varphi}_{m}^{H} \mathbf{F} \tilde{\tilde{\mathbf{D}}}_{m, i} \mathbf{F}^{H} \boldsymbol{\varphi}_{i}=\left\{\begin{array}{l}j \mathbf{I}+\Re\left\{\tilde{\tilde{\mathbf{Q}}}_{m, m}\right\} \text { for } i=m \\ \Re\left\{\tilde{\tilde{\mathbf{Q}}}_{m, i}\right\} \text { for } i \neq m\end{array}\right.$

and

$$
\overline{\tilde{\mathbf{Q}}}_{m, i}=\boldsymbol{\varphi}_{m}^{H} \mathbf{F} \overline{\tilde{\mathbf{D}}}_{m, i} \mathbf{F}^{H} \boldsymbol{\varphi}_{i} \in \mathbb{R}^{N \times N}
$$

6) Combining Real and Imaginary Branches: With (49) and (50), we can derive an estimation of $\mathbf{a}_{m}$ as

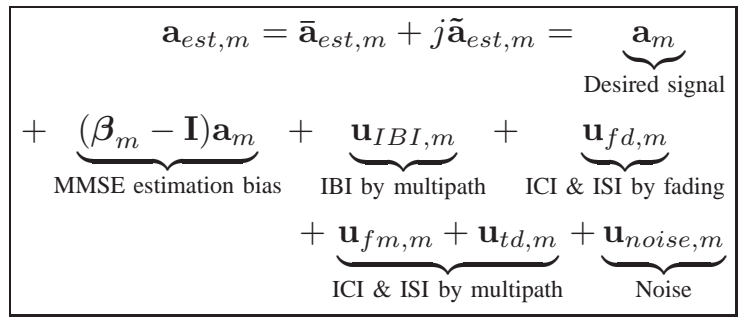

where

$$
\begin{aligned}
& \mathbf{u}_{I B I, m}=\Re\left\{\overline{\mathbf{u}}_{I B I, m}\right\}+j \Im\left\{\tilde{\mathbf{u}}_{I B I, m}\right\} \\
& \mathbf{u}_{f d, m}=\Re\left\{\overline{\mathbf{u}}_{f d, m}\right\}+j \Im\left\{\tilde{\mathbf{u}}_{f d, m}\right\} \\
& \mathbf{u}_{f m, m}=\Re\left\{\overline{\mathbf{u}}_{f m, m}\right\}+j \Im\left\{\tilde{\mathbf{u}}_{f m, m}\right\} \\
& \mathbf{u}_{t d, m}=\Re\left\{\overline{\mathbf{u}}_{t d, m}\right\}+j \Im\left\{\tilde{\mathbf{u}}_{t d, m}\right\} \\
& \mathbf{u}_{\text {noise }, m}=\Re\left\{\overline{\mathbf{u}}_{\text {noise }, m}\right\}+j \Im\left\{\tilde{\mathbf{u}}_{\text {noise }, m}\right\}
\end{aligned}
$$

Note that the desired signal estimation bias $\left(\boldsymbol{\beta}_{m}-\mathbf{I}\right)$ is an effect of compromising the interference and noise of MMSE equalizer. However, $\left(\boldsymbol{\beta}_{m}-\mathbf{I}\right)=\mathbf{0}$ when $\mathrm{ZF}$ receiver is used.

The error terms $\mathbf{u}_{I B I, m}, \mathbf{u}_{f d, m}, \mathbf{u}_{f m, m}$ and $\mathbf{u}_{t d, m}$ depend on the dispersion of the channel, a large delay spread and/or Doppler spread leads to a larger estimation error. However, as will become evident in the next section, the FBMC system is robust to dispersive channels due to the well-localized prototype filters.

\section{INTERFERENCE MSE ANALYSIS}

According to Eq. (55), the equalized signal is contaminated by 6 interferences/noise terms. Among them, the noise term $\mathbf{u}_{\text {noise, } m}$ is independent of all other terms and interference; the IBI contribution $\mathbf{u}_{I B I, m}$ is independent of all of the other terms as well since the interference comes from the previous FBMC block. The MMSE receiver estimation bias $\mathbf{u}_{\text {bias }, m}=$ $\left(\boldsymbol{\beta}_{m}-\mathbf{I}\right) \mathbf{a}_{m}$ is also independent of others since it is the only term that contains the desired signal. $\mathbf{u}_{f d}$ is a function of $\Delta \mathbf{Z}_{l}$ that is dominating by channel temporal correlation error $e_{m, l}$ (i.e., $\Delta \mathbf{Z}_{2, l}$, see Eq. (25) and after), which is uncorrelated to signals and noise. However, $\mathbf{u}_{f m, m}$ and $\mathbf{u}_{t d, m}$ are functions of channel in time and frequency domain, respectively, which are 
correlated to each other. Then we can write the MSE of the $n$-th modulation symbol estimation in the $m$-th FBMC symbol as

$$
\begin{array}{r}
\gamma_{m, n}=\mathcal{E}\left\|a_{e s t, m, n}-\bar{a}_{m, n}\right\|^{2}=\mathcal{E}\left[\left\|\mathbf{u}_{\text {noise }, m}\right\|_{n}^{2}+\left\|\mathbf{u}_{\text {bias }, m}\right\|_{n}^{2}\right. \\
\left.+\left\|\mathbf{u}_{I B I, m}\right\|_{n}^{2}+\left\|\mathbf{u}_{f d, m}\right\|_{n}^{2}+\left\|\mathbf{u}_{f m, m}+\mathbf{u}_{t d, m}\right\|_{n}^{2}\right]
\end{array}
$$

$\|\mathbf{A}\|_{n}^{2}$ means taking the $n$-th diagonal element of matrix $\|\mathbf{A}\|^{2}=\mathbf{A} \mathbf{A}^{H}$.

\section{A. Variance of noise}

Let us first consider the MSE caused by noise and the derived result can serve as a basis for other derivations. It can be proved that

$$
\gamma_{\text {noise }, m, n}=\mathcal{E}\left\|\mathbf{u}_{\text {noise }, m}\right\|_{n}^{2}=\sigma^{2}\left|W_{m, n}\right|^{2}
$$

Proof: see Appendix B.

Eq. (58) shows that the receiver processing (i.e. filtering, DFT and phase shifting) do not change the noise power.

\section{B. Variance of desired signal estimation bias}

The desired signal estimation bias is a result of compromising the desired signal and the noise power by the MMSE receiver. From Eq. (57) and the definition of $\beta_{m, n}$ in (39), we have

$$
\begin{aligned}
& \gamma_{b i a s, m, n}=\mathcal{E}\left\|\mathbf{u}_{b i a s, m}\right\|_{n}^{2}=\mathcal{E}\left\{\left\|\left(\mathbf{I}-\boldsymbol{\beta}_{m}\right) \mathbf{a}_{m}\right\|_{n}^{2}\right\} \\
& =\varrho^{2}\left\|\left(\mathbf{I}-\boldsymbol{\beta}_{m}\right)\right\|_{n}^{2}=\varrho^{2} \frac{\nu^{2} \sigma^{4}}{\left(\varrho^{2}\left|H_{m, n}\right|^{2}+\nu \sigma^{2}\right)^{2}}
\end{aligned}
$$

Apparently, when ZF receiver is adopted, $\gamma_{b i a s, n}=0$ since $\nu=0$. The total contribution due to the noise and desired signal estimation bias for MMSE receiver can be written as

$$
\gamma_{\text {bias }, m, n}+\gamma_{\text {noise }, m, n}=\frac{\sigma^{2}}{\left|H_{m, n}\right|^{2}+\nu \sigma^{2} / \varrho^{2}}
$$

Substituting $\nu=1$ and $\nu=0$ into (60), which corresponds to MMSE and ZF receivers, respectively. We can see that $\frac{\sigma^{2}}{\left|H_{m, n}\right|^{2}+\nu \sigma^{2} / \varrho^{2}} \leq \frac{\sigma^{2}}{\left|H_{m, n}\right|^{2}}$ with limited $\varrho^{2}$ and non-zero $\sigma^{2}$, i.e. MMSE receiver always outperforms $\mathrm{ZF}$ receiver in the absence of other interference.

Apparently, we have the following relationship between noise and desired signal estimation bias

$$
\xi_{\text {bias }, m, n}=\frac{\gamma_{\text {bias }, m, n}}{\gamma_{\text {noise }, m, n}}=\frac{\nu}{\left|H_{m, n}\right|^{2} S N R}
$$

with $\mathrm{SNR}=\varrho^{2} / \sigma^{2}$ being the input SNR.

\section{Variance of IBI}

Let us consider the IBI due to the lack of guard time. We proved in Appendix $\mathrm{C}$ that

$$
\gamma_{I B I, m, n}=\frac{\varrho^{2}}{4}\left|W_{m, n}\right|^{2} \alpha_{I B I, m, n}
$$

with

$$
\begin{array}{r}
\alpha_{I B I, m, n}=\operatorname{Tr}\left(\overline{\mathbf{G}}_{0}^{H} \mathbf{C}_{h}^{c o r r} \overline{\mathbf{G}}_{0}+\tilde{\mathbf{G}}_{0}^{H} \mathbf{C}_{h}^{c o r r} \tilde{\mathbf{G}}_{0}\right) \\
\mathbf{C}_{h}^{c o r r}=\sum_{l=0}^{L-1} \rho_{l}^{2}\left(\overline{\mathbf{C}}_{(l)} \overline{\mathbf{C}}_{(l)}^{H}+\tilde{\mathbf{C}}_{(l)} \tilde{\mathbf{C}}_{(l)}^{H}\right)
\end{array}
$$

where $\overline{\mathbf{C}}_{(l)}=\left[\overline{\mathbf{C}}_{(\text {last }-l)} ; \mathbf{0}_{(M+K-1) N-l \times M N}\right]$ and $\tilde{\mathbf{C}}_{(l)}=$ $\left[\tilde{\mathbf{C}}_{(\text {last }-l)} ; \mathbf{0}_{(M+K-1) N-l \times M N}\right] ; \overline{\mathbf{C}}_{(\text {last }-l)}$ and $\tilde{\mathbf{C}}_{(\text {last }-l)}$ are matrices that contain the last $l$-th rows of $\overline{\mathbf{C}}$ and $\tilde{\mathbf{C}}$, respectively.

In order to compare with noise contribution, we derive (62) by (58), then

$$
\xi_{I B I, m, n}=\frac{\gamma_{I B I, m, n}}{\gamma_{\text {noise }, m, n}}=\frac{S N R}{4} \alpha_{I B I, m, n}
$$

From (64) we can observe that with a fixed SNR, the MSE contribution by IBI is proportional to $\operatorname{Tr}\left(\overline{\mathbf{G}}_{0}^{H} \mathbf{C}_{h}^{\text {corr }} \overline{\mathbf{G}}_{0}+\right.$ $\left.\tilde{\mathbf{G}}_{0}^{H} \mathbf{C}_{h}^{c o r r} \tilde{\mathbf{G}}_{0}\right)$, which is generally very small even for very harsh channel conditions such as LTE ETU (Extended Typical Urban) channel, e.g., $\gamma_{I B I, m, n}<-78 \mathrm{~dB}$ from simulation results in Section $\mathrm{V}$. Thus, this term could be treated as an interference attenuation factor of FBMC systems comparing with (non-CP) OFDM systems, where the IBI (when $M=1$, it is equivalent to ISI) has a linear relationship with $\rho_{l}^{2}$ without any attenuation (p.137) [27].

\section{Variance of $\mathbf{u}_{f d, m}$}

Now let us consider MSE caused by frequency domain channel dispersion. We can prove that

$$
\gamma_{f d, m, n}=\mathcal{E}\left\|\mathbf{u}_{f d, m}\right\|_{n}^{2}=\frac{\varrho^{2}}{4} \alpha_{f d, m, n}\left|W_{m, n}\right|^{2}
$$

where

$$
\alpha_{f d, m, n}=\Re\left\{\mathbf{f}_{n}\left(\overline{\mathbf{C}}_{m}^{H} \boldsymbol{\psi} \overline{\mathbf{C}}_{m}+\tilde{\mathbf{C}}_{m}^{H} \boldsymbol{\psi} \tilde{\mathbf{C}}_{m}\right) \mathbf{f}_{n}^{H}\right\}
$$

with $\mathbf{f}_{n}$ being the $n$-th row of DFT matrix $\mathbf{F} . \boldsymbol{\psi} \approx$ $\sum_{l=0}^{L-1} \rho_{l}^{2} \varpi \operatorname{diag}\left[\overline{\mathbf{C}}_{e}^{\downarrow l}\left(\overline{\mathbf{C}}_{e}^{\downarrow l}\right)^{H}+\tilde{\mathbf{C}}_{e}^{\downarrow l}\left(\tilde{\mathbf{C}}_{e}^{\downarrow l}\right)^{H}\right]$ and $\varpi=\mathbf{I}-$ $\operatorname{diag}\left[\lambda_{m}^{2} \mathbf{1}_{1 \times N}, \cdots, \lambda_{2}^{2} \mathbf{1}_{1 \times N}, \lambda_{1}^{2} \mathbf{1}_{1 \times N}, \lambda_{0}^{2} \mathbf{1}_{1 \times N}, \lambda_{1}^{2} \mathbf{1}_{1 \times N}\right.$, $\left.\lambda_{2}^{2} \mathbf{1}_{1 \times N}, \cdots, \lambda_{K+M-m-1}^{2} \mathbf{1}_{1 \times N}\right]$.

Proof: see Appendix D.

The interference caused by fading channel depends on both real-valued matrices $\varpi$ and $\operatorname{diag}\left[\overline{\mathbf{C}}_{e}^{\downarrow l}\left(\overline{\mathbf{C}}_{e}^{\downarrow l}\right)^{H}+\tilde{\mathbf{C}}_{e}^{\downarrow l}\left(\tilde{\mathbf{C}}_{e}^{\downarrow l}\right)^{H}\right]$. Among the diagonal elements of the matrix $\operatorname{diag}\left[\overline{\mathbf{C}}_{e}^{\downarrow l}\left(\overline{\mathbf{C}}_{e}^{\downarrow l}\right)^{H_{+}}\right.$ $\left.\tilde{\mathbf{C}}_{e}^{\downarrow l}\left(\tilde{\mathbf{C}}_{e}^{\downarrow l}\right)^{H}\right]$, the $n$-th element has the largest value and drop rapidly for the elements depart from the $n$-th element. However, $\varpi$ has an inverse trend as the matrix $\operatorname{diag}\left[\overline{\mathbf{C}}_{e}^{\downarrow l}\left(\overline{\mathbf{C}}_{e}^{\downarrow l}\right)^{H}+\right.$ $\left.\tilde{\mathbf{C}}_{e}^{\downarrow l}\left(\tilde{\mathbf{C}}_{e}^{\downarrow l}\right)^{H}\right]$. Specifically, the value of $n$-th element of $\varpi$ is equal to zero and increases as the index departs from $n$, which implies that the largest element of $\operatorname{diag}\left[\overline{\mathbf{C}}_{e}^{\downarrow l}\left(\overline{\mathbf{C}}_{e}^{\downarrow l}\right)^{H}+\right.$ $\left.\tilde{\mathbf{C}}_{e}^{\downarrow l}\left(\tilde{\mathbf{C}}_{e}^{\downarrow l}\right)^{H}\right]$ will not contribute any error at all and its adjacent elements will be attenuated by the corresponding element of $\varpi$ significantly. Due to this property, the channel fading impact is limited to the adjacent symbols.

To compare the impact of the factor of $\mathbf{u}_{f d, m}$ with the noise, we divide (65) by (58) yielding

$$
\xi_{f d, m, n}=\frac{\gamma_{f d, m, n}}{\gamma_{\text {noise }, m, n}}=\frac{S N R}{4} \alpha_{f d, m, n}
$$

\section{E. Variance of $\mathbf{u}_{f m, m}$ and $\mathbf{u}_{t d, m}$}

Finally, we derive the interference caused by multipath in Appendix E as

$\gamma_{f m+t d, n}=\mathcal{E}\left\|\mathbf{u}_{f m, m}+\mathbf{u}_{t d, m}\right\|_{n}^{2}=\frac{\varrho^{2}}{2} \alpha_{f m+t d, m, n}\left|W_{m, n}\right|^{2}$ 
with

$$
\alpha_{f m+t d, m, n}=\bar{\alpha}_{f m+t d, m, n}+\tilde{\alpha}_{f m+t d, m, n}
$$

where

$$
\begin{aligned}
& \bar{\alpha}_{f m+t d, m, n}=\left|\Re\left(e^{j \theta_{m, n}}\left(\sum_{i=0}^{M-1} \overline{\overline{\mathbf{q}}}_{m, i, n} \Delta \mathbf{H}_{m, n}+\mathbf{f}_{n} \overline{\mathbf{C}}_{m}^{H} \overline{\mathbf{T}}\right)\right)\right|^{2} \\
& +\left|\Im\left(e^{j \theta_{m, n}}\left(\sum_{i=0}^{M-1} \overline{\tilde{\mathbf{q}}}_{m, i, n} \Delta \mathbf{H}_{m, n}+\mathbf{f}_{n} \tilde{\mathbf{C}}_{m}^{H} \overline{\mathbf{T}}\right)\right)\right|^{2} \\
& \tilde{\alpha}_{f m+t d, m, n}=\left|\Re\left(e^{j \theta_{m, n}}\left(\sum_{i=0}^{M-1} \tilde{\overline{\mathbf{q}}}_{m, i, n} \Delta \mathbf{H}_{m, n}+\mathbf{f}_{n} \overline{\mathbf{C}}_{m}^{H} \tilde{\mathbf{T}}\right)\right)\right|^{2} \\
& +\left|\Im\left(e^{j \theta_{m, n}}\left(\sum_{i=0}^{M-1} \tilde{\tilde{\mathbf{q}}}_{m, i, n} \Delta \mathbf{H}_{m, n}+\mathbf{f}_{n} \tilde{\mathbf{C}}_{m}^{H} \tilde{\mathbf{T}}\right)\right)\right|^{2}
\end{aligned}
$$

with $\overline{\mathbf{T}}=\sum_{l=0}^{L-1} \rho_{l} \Delta \overline{\mathbf{C}}^{\downarrow l}$ and $\tilde{\mathbf{T}}=\sum_{l=0}^{L-1} \rho_{l} \Delta \tilde{\mathbf{C}}^{\downarrow l}, \theta_{m, n}$ is the phase of $W_{m, n}$, i.e. $W_{m, n}=\left|W_{m, n}\right| e^{j \theta_{m, n}}$.

There are two types of errors that are caused by the channel multipath effect, i.e. the channel equalization error due to $\overline{\overline{\mathbf{q}}}_{m, i, n}$ (and $\tilde{\tilde{\mathbf{q}}}_{m, i, n}, \overline{\tilde{\mathbf{q}}}_{m, i, n}$ and $\tilde{\overline{\mathbf{q}}}_{m, i, n}$ ), and filter mismatch error related to $\overline{\mathbf{T}}$ (and also $\tilde{\mathbf{T}}$ ). For the former, the $n$-th element of $\Delta \mathbf{H}_{m, n}$ is equal to zero and the index (i.e., $n$ ) corresponds to the largest value of $\overline{\overline{\mathbf{q}}}_{m, i, n}$ (and $\tilde{\tilde{\mathbf{q}}}_{m, i, n}, \overline{\tilde{\mathbf{q}}}_{m, i, n}$ and $\left.\tilde{\overline{\mathbf{q}}}_{m, i, n}\right)$. In addition, for fixed $n$, larger $|i-n|$ results in larger value of the $i$-th element of $\Delta \mathbf{H}_{m, n}$. On the contrary, the element of $\overline{\mathbf{q}}_{m, i, n}$ shows an inverse trend and its absolute value vanishing rapidly as $|i-n|$ goes up. Therefore, ISI and ICI can be reduced significantly by the attenuation factors $\Delta \overline{\mathbf{C}}$ and $\Delta \mathbf{H}_{m, n}$, which makes the FBMC systems more robust to multipath than OFDM ststems.

To show the effect of the factor of $\mathbf{u}_{t d, m}$ over the noise, we divide (68) by (58), resulting in

$$
\xi_{f m+t d, m, n}=\frac{\gamma_{f m+t d, m, n}}{\gamma_{\text {noise }, m, n}}=\frac{S N R}{2} \alpha_{f m+t d, m, n}
$$

\section{F. Total MSE of FBMC System}

So far, we have derived all the terms listed in (57) one-byone. Substituting (58), (59), (62), (65), (68) into (57), we have the total MSE of the FBMC system in the presence of doubly dispersive channel and noise as follows:

$$
\begin{aligned}
\gamma_{m, n} & =\gamma_{\text {noise }, m, n}+\gamma_{\text {bias }, m, n}+\gamma_{I B I, m, n} \\
& +\gamma_{f d, m, n}+\gamma_{f m+t d, m, n}
\end{aligned}
$$

Using the relationship of each term with noise caused MSE in (61), (64), (67) and (71), we have

$$
\begin{array}{r}
\gamma_{m, n}=\gamma_{\text {noise }, m, n}\left[1+\frac{S N R}{4}\left(\alpha_{I B I, m, n}+\alpha_{f d, m, n}\right.\right. \\
\left.\left.+2 \alpha_{f m+t d, m, n}\right)+\frac{\nu}{S N R\left|H_{m, n}\right|^{2}}\right]
\end{array}
$$

Comparing with the MSE caused by noise only, Eq. (73) reveals that the errors caused by the doubly dispersive channel (also desired signal estimation bias for MMSE receiver) will increase the total MSE by a factor of $\left[1+S N R / 4\left(\alpha_{f d, m, n}+\right.\right.$ $\left.\left.\alpha_{I B I, m, n}+\alpha_{f m+t d, m, n}\right)+\nu / S N R /\left|H_{m, n}\right|^{2}\right]$. The result implies that for a given MSE caused by noise, we can estimate the total MSE of the system, which provides a theoretical guideline to design a FBMC systems. More precisely, one can obtain the MSE caused by the noise as a baseline, then derive the total MSE analytically by considering the parameters such as Doppler spread, delay spread, filter parameters.

For any given specific channel and Doppler spread, one can always analytically calculate the interference caused by the channel dispersions by Eq. (73), based upon which we can determine whether the FBMC system under a certain channel condition will result in a negligible or significant ICI/ISI/IBI in comparison to the error caused only by the noise.

\section{G. SINR of FBMC System}

With the given estimation MSE $\gamma_{m, n}$ and normalized desired signal power, we can readily express the SINR of the FBMC system in the doubly dispersive channel as

$$
S I N R_{m, n}=\frac{1}{\gamma_{m, n}}
$$

\section{NUMERICAL RESULTS}

In this section, we use Monte-Carlo simulations to compare the simulated MSE to the analytical and examine the effects of different contributing factors on the system performance in various time and frequency dispersive channels. We adopt the LTE/LTE-A defined radio frame structure, i.e. $20 \mathrm{MHz}$ bandwidth containing 1200 subcarriers with subcarrier spacing $\Delta f=15 \mathrm{KHz}$ and the symbol duration $\Delta T=1 / 15000 \mathrm{~s}$. The three radio channels: EPA (Extended Pedestrian-A)/EVA (Extended Vehicular-A)/ETU channels used in LTE/LTE-A are adopted in our simulations [28]. Note that the delay spread of these three channels are fixed, which are $\tau_{R M S}=43 \mathrm{~ns}, 357$ ns, $991 \mathrm{~ns}$, respectively. In order to investigate the impact of the continuously changing $\tau_{R M S}$ on the FBMC system, we will later use IEEE 802.11 radio channel models with variable length of delay spread [29]. The Doppler spread also varies to illustrate the impact of channel time domain dispersion on the FBMC system. For the FBMC specified parameters: the IOTA (isotropic orthogonal transform algorithm) prototype filter is adopted in our simulations [15] with overlapping factor $K=6$ for most simulations. However, simulation results with smaller overlapping factor $K=4$ will also be shown for comparison purposes. The desired signal is modulated by QPSK (Quadrature Phase Shift Keying) with normalized power and the input SNR is controlled by the noise power. Since MMSE and ZF equalizers show similar trend and the former one is more generic, therefore, we only present results for the MMSE-based algorithm in our simulations.

1) Impact of frequency domain dispersive channel: To investigate the effect of Doppler spread, we plot the MSE versus to SNR values with various $f_{D}$ in the LTE EPA channel. $f_{D}$ changes in the range $[10,50,100,300,600] \mathrm{Hz}$, which correspond to $[5.4,27,54,162,324] \mathrm{km} / \mathrm{h}$ moving speed at $2 \mathrm{GHz}$ carrier frequency, or $[2.2,10.8,21.6,64.8,130] \mathrm{km} / \mathrm{h}$ moving speed at $5 \mathrm{GHz}$ carrier frequency. From Fig. 2, we can see that in all cases, the analytical results match the simulation results nearly perfect. In addition, interference floors are shown 
for different values of $f_{D}$ and it can be seen that a larger $f_{D}$ leads to a higher floor. As an extreme case, the MSE can reach $-13 \mathrm{~dB}$ with $f_{D}=600 \mathrm{~Hz}$ at $\mathrm{SNR}=30 \mathrm{~dB}$ in EPA channel.

Note that as the SNR increases, the output MSE decreases first and then goes up slightly, e.g., when $f_{D}=600 \mathrm{~Hz}$, the MSE achieves a minimum value at $\mathrm{SNR}=30 \mathrm{~dB}$ and goes up when SNR larger than $30 \mathrm{~dB}$, which is due to the reason that in the high SNR region, the total MSE of the estimated symbol is dominatingly contributed by the interference instead of noise and the desired signal estimation bias, i.e., we can rewrite (73) as $\gamma_{m, n} \approx \frac{1}{4}\left|W_{m, n}\right|^{2} \varrho^{2}\left(\alpha_{I B I, m, n}+\alpha_{f d, m, n}+2 \alpha_{f m+t d, m, n}\right)$, by considering MMSE equalizer defined in (36) and fixed signal power $\varrho^{2}=1$, we then have $\left|W_{m, n}\right|^{2}=\frac{\left|H_{m, n}\right|^{2}}{\left(\left|H_{m, n}\right|^{2}+\sigma^{2}\right)^{2}}$. In the frequency selective channel environments, the relatively larger noise power $\sigma^{2}$ can significantly reduce $\left|W_{m, n}\right|^{2}$ in the deep fading subcarriers (i.e., $\left|H_{m, n}\right| \approx 0$ ). In other words, the smaller $\sigma^{2}$ (i.e., higher SNR) leads to larger scaling factor $\left|W_{m, n}\right|^{2}$, as a result, the interference will be amplified significantly and contributes more MSE than the reduction of the contribution from noise and desired signal estimation bias in a higher SNR value.

Note that the simulation results for $f_{D}=10 \mathrm{~Hz}$ with $K=4$ are also shown in Fig. 2 for comparison purposes. It can be seen that the error floors caused by both channel and prototype filter in this case (EPA channel with $f_{D}=10 \mathrm{~Hz}$ ) are $-42 \mathrm{~dB}$ and $-40.5 \mathrm{~dB}$ for $K=6$ and $K=4$, respectively. In other words, the finite length prototype filter caused interference is less than $-42 \mathrm{~dB}$ and $-40.5 \mathrm{~dB}$ for $K=6$ and $K=4$, respectively. With a larger Doppler shift (e.g., from $f_{D}=10$ $\mathrm{Hz}$ to $f_{D}=50 \mathrm{~Hz}$ ), the doubly dispersive channel caused errors tend to be dominant, rendering the prototype filter caused interference to be negligible and the curves for $K=6$ completely overlap with $K=4$, which are not shown in the figure for brevity.

The effect of various contributing factors contributing to MSE listed in Eq. (72) (or Eq. (73)) are shown in Fig. 4 where the MSE caused by noise goes down linearly as the SNR increases, and it can be seen that the interference caused by IBI in the EPA channel is negligible $(<-88 \mathrm{~dB})$, while EPA channel multipath effect (e.g. $\gamma_{f m+t d}$ ) can be more significant than noise when SNR $>53 \mathrm{~dB}$. Otherwise, it remains negligible compared to noise. While the effect of channel fading depends on $f_{D}$, for example, when $f_{D}=600$ $\mathrm{Hz}$, the $\gamma_{f d}$ becomes dominating for SNR $>15 \mathrm{~dB}$, which will affect symbol detection in high modulation level.

In addition to MSE, the output SINR versus to symbol input SNR values with various $f_{D}$ in the LTE EPA channel is shown in the left-hand side subplot in Fig. 3. Again, the analytical results match the simulation results nearly perfectly for all cases. The reason that the output SINR values increases first and then goes down slightly is the same as for MSE performance shown in Fig. 2.

2) Impact of time domain dispersion: The multipath effect of the channel is shown in Fig. 5 with Doppler spread $f_{D}=0$ $\mathrm{Hz}$, where we used the EPA, EVA and ETU channels. Again, the simulated and analytical curves concur with each other. As the delay spread increases, the error floor due to the channel multipath effect (especially, $\mathbf{u}_{f m}$ and $\mathbf{u}_{f d}$ ) goes up

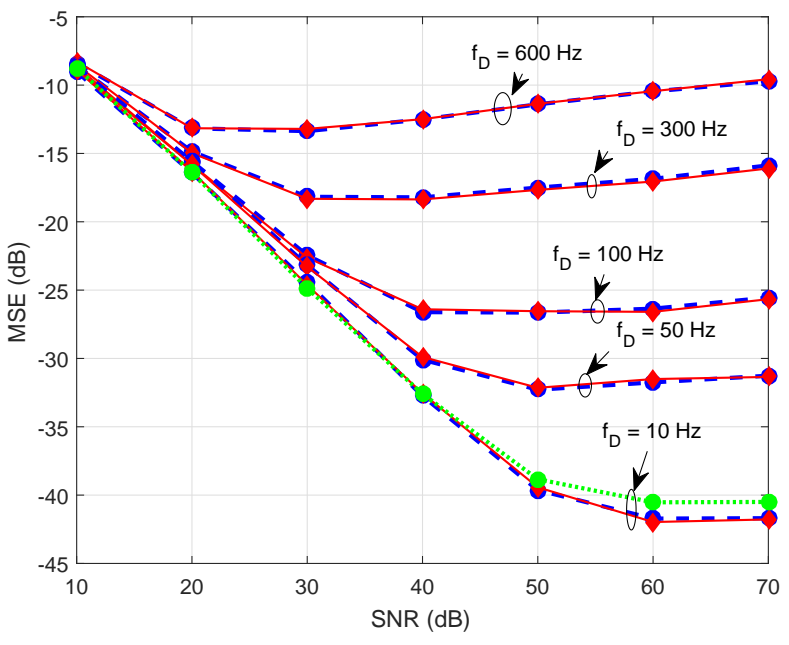

Fig. 2. MSE under EPA channel and various Doppler spread $f_{D}$, solid lines and dashed lines are for the analytical and simulated results with $K=6$, respectively, dotted line is the simulated results with $K=4$.
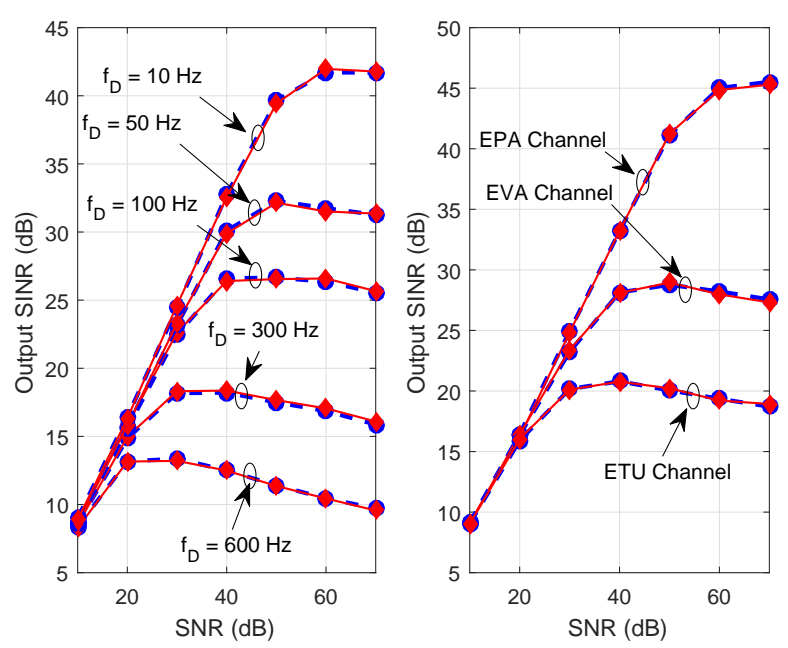

Fig. 3. Output SINR versus symbol input SNR under various Doppler spread $f_{D}$ and channels. Solid lines and dashed lines are for the analytical and simulated results, respectively. Left: output SINR versus input SNR under various Doppler spread $f_{D}$ in EPA channel; Right: output SINR versus input SNR under various channels with Doppler spread $f_{D}=0 \mathrm{~Hz}$.

accordingly. In the ETU channel, the MSE can reach as high as $-20 \mathrm{~dB}$.

We also provide the simulation results for $K=4$ in EPA channel in Fig. 5. Again, from the figure we can see that the insufficient overlapping factor caused errors are smaller than $-45 \mathrm{~dB}$ and $-42 \mathrm{~dB}$ for $K=6$ and $K=4$, respectively, which are negligible for most of the wireless communication systems. With a more harsh channel (e.g., from EPA to EVA), the doubly dispersive channel caused error tends to be dominant, making the prototype filter caused interference negligible and the curves for $K=6$ are totally overlapped with $K=4$, which are not shown in the figure for brevity.

The effect of the contributing factors $\gamma_{I B I}, \gamma_{f m+t d}, \gamma_{\text {noise }}$, $\gamma_{\text {bias }}\left(\gamma_{f d}=0\right.$ since $\left.f_{D}=0\right)$ are shown in Fig. 6 for the 


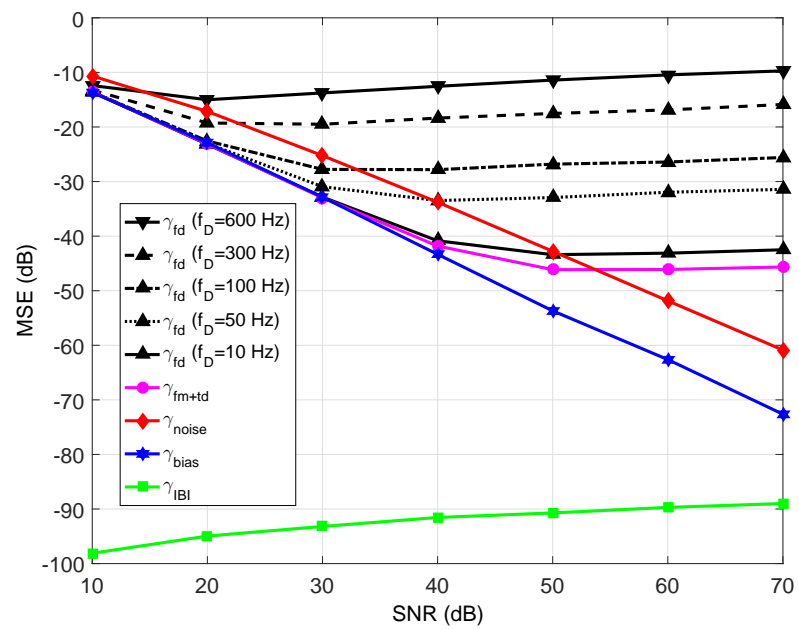

Fig. 4. MSE contributing factors under EPA channel and various Doppler spread $f_{D}$.

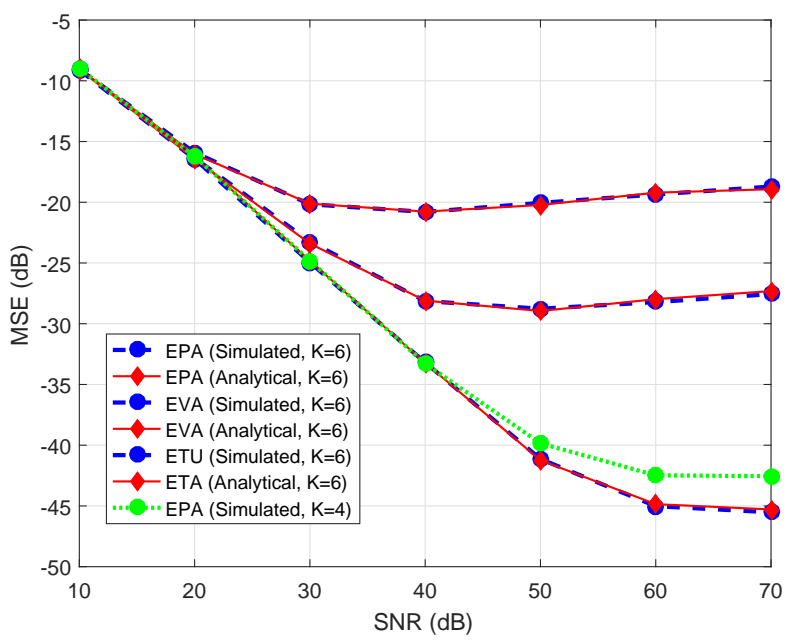

Fig. 5. MSE versus SNR under EPA/EVA/ETU channels with $f_{D}=0$.

ETU channel, where the IBI contributed MSE is negligible, however, comparing to the curve shown in Fig. 4, where the IBI generates $-98 \mathrm{~dB}$ MSE at SNR $=10 \mathrm{~dB}$ in the EPA channel, it rises to $-88 \mathrm{~dB}$ in the ETU channel at the same SNR. The factor $\gamma_{f m+t d}$ can create relatively larger interference than noise when SNR $>25 \mathrm{~dB}$. Again, the output MSE decreases first and then goes up slightly, which is again due to the behavior of MMSE equalizer in the presence of interference as explained in the last simulation.

The output SINR versus to symbol input SNR with various channel with $f_{D}=0 \mathrm{~Hz}$ is shown in the right-hand side subplot in Fig. 3. It can be seen that the analytical results match the simulation results nearly perfectly for all channels.

3) Impact of doubly dispersive channel: Next, we assume both Doppler spread and delay spread as variables and examine the system performance in different channel conditions. In order to show the impact of continuously changing delay spread on the FBMC system, we adopt the

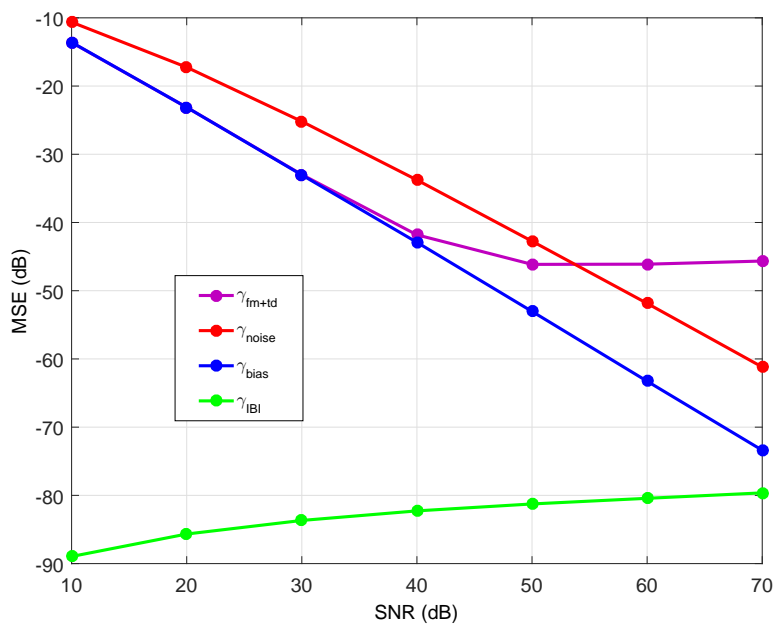

Fig. 6. MSE contributing factors in ETU channel with $f_{D}=0$.

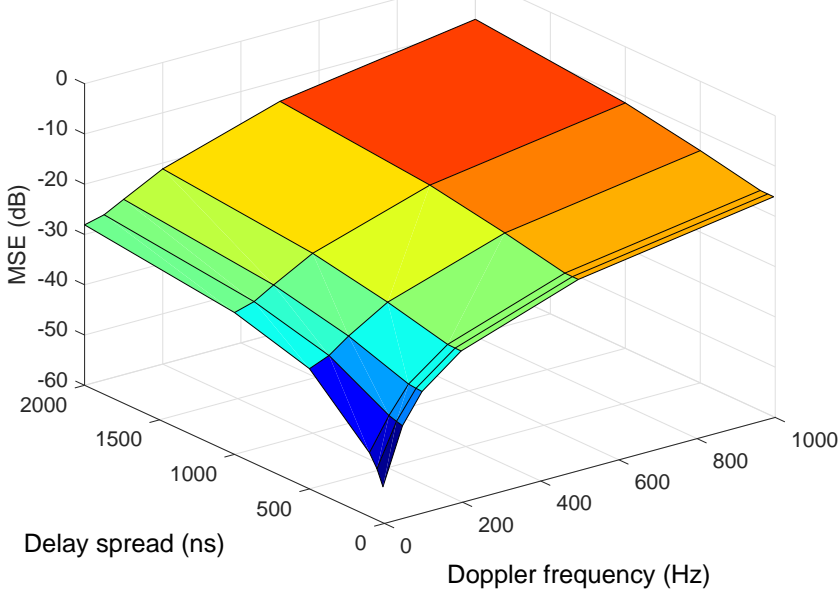

Fig. 7. MSE under various Doppler spread $f_{D}$ and delay spread $\tau_{R M S}$.

IEEE 802.11 channel model with the delay spread $\tau_{R M S}=$ $[10,50,100,200,500,1000] \mathrm{ns}$ and sampling frequency 30.72 MHz. Note that due to the very close agreement between simulation and analytical results, we will only show analytical results in Fig. 7 for high SNR region at SNR $=50 \mathrm{~dB}$ to focus on the interference only. From the 3D figure we can see that the joint impact of both time and frequency domain channel dispersion can bring significant performance degradation.

4) Optimal system design: For a given channel, a reduction on subcarrier spacing $\Delta f$ can reduce the impact of multipath, however, it will certainly increase symbol duration $\Delta T$ since $\Delta T \Delta f=1$ to maintain the real-domain orthogonality and leads to a larger impact by Doppler spread. Thus, the optimal radio frame design should adapt to the specific channel conditions to minimize the total MSE caused by doubly dispersive channels.

Fig. 8 shows the optimal symbol duration of the FBMC system for various Doppler spread and delay spread, where 




Fig. 8. Optimal symbol duration (normalized by $\Delta T$ ) under various Doppler spread $f_{D}$ and delat spread $\tau_{R M S}$.

the optimal symbol duration is normalized by LTE symbol duration (i.e., $\Delta T=1 / 15000 \mathrm{~s}$ ), e.g. an optimal value equal to 0.4 implies that the optimal symbol duration is $0.4 \Delta T$. From the figure it can be seen that a larger $f_{D}$ leads to a smaller optimal symbol duration to mitigate the channel frequency domain dispersion effect. A larger $\tau_{R M S}$, however, will lead to a richer multipath channel, requires a smaller subcarrier spacing (therefore, a larger symbol duration) to mitigate the channel frequency selectivity. Note that the corresponding optimal values will change when advanced multi-tap equalization algorithms are adopted.

\section{CONClusions AND Future WORKS}

The impact of doubly dispersive channels on FBMC systems has been analyzed in terms of MSE, for both MMSE and ZF-based one-tap channel equalization algorithms. The contributing interference and noise factors have been derived individually under our analytical framework. We first proved that the circular convolution property between the symbols and corresponding channel coefficients holds for the FBMC system by adding a set of inaccuracies, whose values are given analytically. The quantitative analysis helps identify whether each error term is negligible or not for given radio channel. Our theoretical analysis has been validated by simulations. In addition, the results reveal that with extremely large Doppler spread or channel delay spread, the FBMC system performance may be severely be limited by strong interference. In such cases, we need to resort to more complex multitap equalization schemes, rather than one-tap equalization. The analytical framework developed in this paper provides a valuable reference for the design and development of practical FBMC systems.

Future work can be focused on the following topics: 1) the prototype filter caused interference (especially with small overlapping factor) can be taken into consideration; 2) the analytical bit error rate for the FBMC system in the presence of doubly dispersive channel could be another metric for the performance analysis.

\section{ACKNOWLEDGEMENT}

The author would like to acknowledge the support of the University of Surrey 5GIC (http://www.surrey.ac.uk/5gic) members for this work.

\section{APPENDIX A}

PROOF OF EQ. (33)

According to (20) and (21), we can write $\sum_{l=0}^{L-1} \rho_{l} z_{m, l} \overline{\mathbf{x}}_{e, i}^{\downarrow l}=\overline{\mathbf{X}}_{c i r, i} \mathbf{h}_{m}$ with $\overline{\mathbf{X}}_{c i r, i}=\left[\overline{\mathbf{x}}_{e, i}^{\downarrow 0}, \overline{\mathbf{x}}_{e, i}^{\downarrow 1} \cdots\right.$,

$\left.\overline{\mathbf{x}}_{e, i}^{\downarrow L-1}\right]$ being a circular convolution matrix. We can express $\overline{\mathbf{u}}_{R, m}$ as

$$
\begin{aligned}
\overline{\mathbf{u}}_{R, m} & =\mathbf{W}_{m} \boldsymbol{\varphi}_{m}^{H} \mathbf{F}\left[\sum_{i=0}^{M-1} \overline{\overline{\mathbf{D}}}_{m, i} \overline{\mathbf{X}}_{c i r, i} \mathbf{h}_{m}\right] \\
& =\mathbf{W}_{m} \boldsymbol{\varphi}_{m}^{H} \mathbf{F} \sum_{i=0}^{M-1} \overline{\overline{\mathbf{D}}}_{m, i} \mathbf{F}^{H} \mathbf{F} \overline{\mathbf{X}}_{c i r, i} \mathbf{F}^{H} \mathbf{F} \mathbf{h}_{m}
\end{aligned}
$$

where we used $\mathbf{F}^{H} \mathbf{F}=\mathbf{I}$. Then we can use the circular convolution property as follows (pp. 129-130) [27]

$$
\mathbf{F} \overline{\mathbf{X}}_{c i r, i} \mathbf{F}^{H} \mathbf{F} \mathbf{h}_{m}=\mathbf{H}_{m}\left(\mathbf{F} \overline{\mathbf{x}}_{i}\right)
$$

with $\overline{\mathbf{x}}_{i}$ being the first column of $\overline{\mathbf{X}}_{c i r, i}$. And $\mathbf{F} \overline{\mathbf{x}}_{i}$ denotes the DFT processing of $\overline{\mathbf{x}}_{i}$ and according to (10) and (9), we have $\mathbf{F} \overline{\mathbf{x}}_{i}=\overline{\mathbf{b}}_{i}=\varphi_{i} \overline{\mathbf{a}}_{i}$, substituting it into (76) and (75) leads to

$$
\overline{\mathbf{u}}_{R, m}=\sum_{i=0}^{M-1} \mathbf{W}_{m} \boldsymbol{\varphi}_{m}^{H} \mathbf{F} \overline{\overline{\mathbf{D}}}_{m, i} \mathbf{F}^{H} \mathbf{H}_{m} \boldsymbol{\varphi}_{i} \overline{\mathbf{a}}_{i}
$$

The order of $\mathbf{H}_{m}$ and $\boldsymbol{\varphi}_{i}$ are exchangeable since both are diagonal, we can thus obtain (33).

\section{APPENDIX B}

ProOF OF EQ. (58)

From Eq. (56), we can write

$$
\begin{aligned}
& \gamma_{\text {noise }, m}=\mathcal{E}\left\|\Re\left\{\overline{\mathbf{u}}_{\text {noise }, m}\right\}+j \Im\left\{\tilde{\mathbf{u}}_{\text {noise }, m}\right\}\right\|^{2} \\
& =\frac{1}{4} \mathcal{E}\left\|\left(\overline{\mathbf{u}}_{\text {noise }, m}+\tilde{\mathbf{u}}_{\text {noise }, m}\right)+\left(\overline{\mathbf{u}}_{\text {noise }, m}^{*}-\tilde{\mathbf{u}}_{\text {noise }, m}^{*}\right)\right\|^{2} \\
& =\frac{1}{4}\left[\mathcal{E}\left\|\overline{\mathbf{u}}_{\text {noise }, m}+\tilde{\mathbf{u}}_{\text {noise } m}\right\|^{2}+\mathcal{E}\left\|\overline{\mathbf{u}}_{\text {noise }, m}^{*}-\tilde{\mathbf{u}}_{\text {noise }, m}^{*}\right\|^{2}\right]
\end{aligned}
$$

$\left(\overline{\mathbf{u}}_{\text {noise }, m}+\tilde{\mathbf{u}}_{\text {noise }, m}\right)$ and $\left(\overline{\mathbf{u}}_{\text {noise }, m}^{*}-\tilde{\mathbf{u}}_{\text {noise }, m}^{*}\right)$ are uncorrelated under the assumption that the noise is proper [30]. By using the last term of (32) and the last Eq. in (51), we have

$$
\begin{aligned}
& \gamma_{\text {noise }, m}=\frac{1}{4} \Re\left\{\mathcal { E } \left[\mathbf { W } _ { m } \boldsymbol { \varphi } _ { m } ^ { H } \mathbf { F } \left(\overline{\mathbf{C}}_{m}^{H} \mathbf{n} \mathbf{n}^{H} \overline{\mathbf{C}}_{m}+\tilde{\mathbf{C}}_{m}^{H} \mathbf{n} \mathbf{n}^{H} \tilde{\mathbf{C}}_{m}\right.\right.\right. \\
& \left.\left.\left.+\overline{\mathbf{C}}_{m}^{H} \mathbf{n} \mathbf{n}^{H} \overline{\mathbf{C}}_{m}+\tilde{\mathbf{C}}_{m}^{H} \mathbf{n} \mathbf{n}^{H} \tilde{\mathbf{C}}_{m}\right) \mathbf{F}^{H} \boldsymbol{\varphi}_{m} \mathbf{W}_{m}^{H}\right]\right\} \\
& =\frac{\sigma^{2}}{2} \Re\left\{\mathbf{W}_{m} \boldsymbol{\varphi}_{m}^{H} \mathbf{F} \sum_{i=0}^{M}\left(\overline{\overline{\mathbf{D}}}_{m, i}-j \tilde{\tilde{\mathbf{D}}}_{m, i}\right) \mathbf{F}^{H} \boldsymbol{\varphi}_{m} \mathbf{W}_{m}^{H}\right\}
\end{aligned}
$$

According to (34) and (53), $\quad \sum_{i=0}^{M-1} \overline{\overline{\mathbf{Q}}}_{m, i}=$ $\mathbf{I}+\Im\left\{\sum_{i=0}^{M-1} \overline{\overline{\mathbf{Q}}}_{m, i}\right\} \quad$ and $\quad-j \sum_{i=0}^{M-1} \tilde{\tilde{\mathbf{Q}}}_{m, i}=$ $\left.\mathbf{I}-\Im\left\{\sum_{i=0}^{M-1} \tilde{\tilde{\mathbf{Q}}}_{m, i}\right\}\right)$, i.e. $\Re\left\{\sum_{i=0}^{M-1}\left(\overline{\overline{\mathbf{Q}}}_{m, i}+\tilde{\overline{\mathbf{Q}}}_{m, i}\right)\right\}=2 \mathbf{I}$, substituting it into (79) leads to $\gamma_{\text {noise }, m}=\sigma^{2} \mathcal{E}\left(\mathbf{W}_{m} \mathbf{W}_{m}^{H}\right)$. Taking the $n$-th diagonal element of $\gamma_{\text {noise, } m}$, we obtain (58). 


\section{APPENDiX C}

PROOF OF EQ. (62)

Using Eq. (56) and following the same derivation as in (78) for the noise, we have

$$
\begin{aligned}
\gamma_{I B I, m} & =\mathcal{E}\left\|\Re\left\{\overline{\mathbf{u}}_{I B I, m}\right\}+j \Im\left\{\tilde{\mathbf{u}}_{I B I, m}\right\}\right\|^{2} \\
& =\frac{1}{2} \Re\left\{\mathcal{E}\left\|\overline{\mathbf{u}}_{I B I, m}+j \tilde{\mathbf{u}}_{I B I, m}\right\|^{2}\right\}
\end{aligned}
$$

With the definitions of $\overline{\mathbf{u}}_{I B I, m}$ and $\tilde{\mathbf{u}}_{I B I, m}$ in the first term of (32) and the first Eq. in (51), then

$\gamma_{I B I, m}=\frac{1}{2} \Re\left\{\mathcal{E} \| \mathbf{W}_{m} \boldsymbol{\varphi}_{m}^{H} \mathbf{F}\left[\left(\overline{\mathbf{C}}_{m}+j \tilde{\mathbf{C}}_{m}\right) \mathbf{v}_{I B I, m} \|^{2}\right\}\right.$

$=\frac{\varrho^{2}}{4} \Re\left\{\mathbf{W}_{m} \boldsymbol{\varphi}_{m}^{H} \mathbf{F}\left(\overline{\mathbf{C}}_{m}+j \tilde{\mathbf{C}}_{m}\right) \boldsymbol{\psi}_{I B I}\left(\overline{\mathbf{C}}_{m}-j \tilde{\mathbf{C}}_{m}\right)^{H} \mathbf{F}^{H} \boldsymbol{\varphi}_{m} \mathbf{W}_{m}^{H}\right\}(81)$

where $\psi_{I B I}=\mathcal{E}\left\{\mathbf{v}_{I B I} \mathbf{v}_{I B I}^{H}\right\}$. From (16) we have $\psi_{I B I}=$ $\mathcal{E}\left\{\sum_{l=0}^{L-1} \rho_{l}^{2} \mathbf{Z}_{l} \mathcal{E}\left(\mathbf{y}_{B, l} \mathbf{y}_{B, l}^{H}\right) \mathbf{Z}_{l}^{H}\right\}$, where the factors $\mathbf{Z}_{l}$ and $\mathbf{y}_{B, l}$ are uncorrelated. $\mathcal{E}\left(\mathbf{y}_{B, l} \mathbf{y}_{B, l}^{H}\right)$ is dependent on the signal type of the last block, where we assume it is also occupied by an FBMC symbol with the same power, then we have $\mathcal{E}\left(\mathbf{y}_{B, l} \mathbf{y}_{B, l}^{H}\right)=\overline{\mathbf{C}}_{(l)} \mathcal{E}\left\{\overline{\mathbf{x}}_{\text {last }} \overline{\mathbf{x}}_{\text {last }}^{H}\right\} \overline{\mathbf{C}}_{(l)}^{H}+$ $\tilde{\mathbf{C}}_{(l)} \mathcal{E}\left\{\tilde{\mathbf{x}}_{\text {last }} \tilde{\mathbf{x}}_{\text {last }}^{H}\right\} \tilde{\mathbf{C}}_{(l)}^{H}, \overline{\mathbf{x}}_{\text {last }}$ and $\tilde{\mathbf{x}}_{\text {last }}$ are the real and imaginary parts of symbols (after IDFT and phase shifting) in the last block, and $\overline{\mathbf{x}}_{\text {last }}$ and $\tilde{\mathbf{x}}_{\text {last }}$ are uncorrelated to each other. Note that $\overline{\mathbf{x}}_{\text {last }}$ and $\tilde{\mathbf{x}}_{\text {last }}$ have the same definition as (10) and (13). It is easy to prove that $\mathcal{E}\left\{\overline{\mathbf{x}}_{\text {last }} \overline{\mathbf{x}}_{\text {last }}^{H}\right\}=$ $\mathcal{E}\left\{\tilde{\mathbf{x}}_{\text {last }} \tilde{\mathbf{x}}_{\text {last }}^{H}\right\}=\varrho^{2} / 2 \mathbf{I}$. Then we have $\mathcal{E}\left(\mathbf{y}_{B, l} \mathbf{y}_{B, l}^{H}\right)=$ $\frac{\varrho^{2}}{2}\left(\overline{\mathbf{C}}_{(l)} \overline{\mathbf{C}}_{(l)}^{H}+\tilde{\mathbf{C}}_{(l)} \tilde{\mathbf{C}}_{(l)}^{H}\right)=\frac{\varrho^{2}}{2} \mathbf{C}_{(l)}^{c o r r}$, where $\mathbf{C}_{(l)}^{c o r r}$ is a diagonal matrix with the first $l$-th diagonal elements being nonzero and $\mathcal{E}\left\{\sum_{l=0}^{L-1} \rho_{l}^{2} \mathbf{Z}_{l} \mathbf{C}_{(l)}^{c o r r} \mathbf{Z}_{l}^{H}\right\}=\sum_{l=0}^{L-1} \rho_{l}^{2} \mathbf{C}_{(l)}^{\text {corr }}$. Then we have $\boldsymbol{\psi}_{I B I}=\frac{\varrho^{2}}{2} \mathcal{E}\left\{\sum_{l=0}^{L-1} \rho_{l}^{2} \mathbf{Z}_{l} \mathbf{C}_{(l)}^{\text {corr }} \mathbf{Z}_{l}^{H}\right\}=\frac{\varrho^{2}}{2} \mathbf{C}_{h}^{\text {corr }}$, substituting it into (81), yields

$$
\begin{array}{r}
\gamma_{I B I, m}=\frac{\varrho^{2}}{4} \Re\left[\mathbf { W } _ { m } \boldsymbol { \varphi } _ { m } ^ { H } \mathbf { F } \left(\overline{\mathbf{G}}_{0}^{H} \mathbf{C}_{h}^{c o r r} \overline{\mathbf{G}}_{0}-j \overline{\mathbf{G}}_{0}^{H} \mathbf{C}_{h}^{c o r r} \tilde{\mathbf{G}}_{0}\right.\right. \\
\left.\left.-j \tilde{\mathbf{G}}_{0}^{H} \mathbf{C}_{h}^{c o r r} \overline{\mathbf{G}}_{0}+\tilde{\mathbf{G}}_{0}^{H} \mathbf{C}_{h}^{c o r r} \tilde{\mathbf{G}}_{0}\right) \mathbf{F}^{H} \boldsymbol{\varphi}_{m} \mathbf{W}_{m}^{H}\right]
\end{array}
$$

Note that $\overline{\mathbf{G}}_{0}^{H} \mathbf{C}_{h}^{c o r r} \overline{\mathbf{G}}_{0}, \quad \tilde{\mathbf{G}}_{0}^{H} \mathbf{C}_{h}^{c o r r} \tilde{\mathbf{G}}_{0}, \overline{\mathbf{G}}_{0}^{H} \mathbf{C}_{h}^{c o r r} \tilde{\mathbf{G}}_{0}$ and $\tilde{\mathbf{G}}_{0}^{H} \mathbf{C}_{h}^{c o r r} \overline{\mathbf{G}}_{0}$, are real-valued diagonal matrices. By taking the $n$-th element of $\gamma_{I B I}$, we can obtain (62).

\section{APPENDIX D}

PROOF OF EQ. (65)

Using Eq. (56) and following the same derivation in (78) for noise MSE, we have

$$
\begin{aligned}
\gamma_{f d, m} & =\mathcal{E}\left\|\Re\left\{\overline{\mathbf{u}}_{f d, m}\right\}+j \Im\left\{\tilde{\mathbf{u}}_{f d, m}\right\}\right\|^{2} \\
& =\frac{1}{2} \Re\left\{\mathcal{E}\left\|\overline{\mathbf{u}}_{f d, m}+j \tilde{\mathbf{u}}_{f d, m}\right\|^{2}\right\}
\end{aligned}
$$

With the definitions of $\overline{\mathbf{u}}_{f d, m}$ and $\tilde{\mathbf{u}}_{f d, m}$ in the fourth term of (32) and third Eq. of (51) respectively, and further notice the definition of $\mathbf{v}_{f d, m}$ in (28), we have

$$
\begin{array}{r}
\gamma_{f d, m}=\frac{1}{2} \Re\left\{\mathcal{E} \| \mathbf{W}_{m} \boldsymbol{\varphi}_{m}^{H} \mathbf{F}\left[\left(\overline{\mathbf{C}}_{m}+j \tilde{\mathbf{C}}_{m}\right) \mathbf{v}_{f d} \|^{2}\right\}\right. \\
=\frac{\varrho^{2}}{4} \Re\left\{\mathbf{W}_{m} \boldsymbol{\varphi}_{m}^{H} \mathbf{F}\left(\overline{\mathbf{C}}_{m}+j \tilde{\mathbf{C}}_{m}\right) \psi\left(\overline{\mathbf{C}}_{m}-j \tilde{\mathbf{C}}_{m}\right)^{H} \mathbf{F}^{H} \boldsymbol{\varphi}_{m} \mathbf{W}_{m}^{H}\right\}
\end{array}
$$

where $\boldsymbol{\psi}=\mathcal{E}\left\{\mathbf{v}_{f d} \mathbf{v}_{f d}^{H}\right\} \frac{2}{\rho^{2}}=\mathcal{E}\left\{\left[\sum_{l=0}^{L-1} \rho_{l} \Delta \mathbf{Z}_{l}\left(\overline{\mathbf{C}}_{e}^{\downarrow l} \overline{\mathbf{x}}_{e}^{\downarrow l}+\right.\right.\right.$ $\left.\left.\left.\tilde{\mathbf{C}}_{e}^{\downarrow l} \tilde{\mathbf{x}}_{e}^{\downarrow l}\right)\right]\left[\sum_{l=0}^{L-1} \rho_{l} \Delta \mathbf{Z}_{l}\left(\overline{\mathbf{C}}_{e}^{\downarrow l} \overline{\mathbf{x}}_{e}^{\downarrow l}+\tilde{\mathbf{C}}_{e}^{\downarrow l} \tilde{\mathbf{x}}_{e}^{\downarrow l}\right)\right]^{H}\right\}$. By using $\Delta \mathbf{Z}_{l} \approx \Delta \mathbf{Z}_{2, l}$ (see Eq. (25) and after) and the fact that $\tilde{\mathbf{x}}_{e}^{\downarrow l}$ and $\overline{\mathbf{x}}_{e}^{\Downarrow l}$ are independent, we have $\boldsymbol{\psi} \approx$ $\mathcal{E}\left\{\sum_{l=0}^{L-1} \rho_{l}^{2} \Delta \mathbf{Z}_{2, l}\left[\overline{\mathbf{C}}_{e}^{\downarrow l}\left(\overline{\mathbf{C}}_{e}^{\downarrow l}\right)^{H}+\tilde{\mathbf{C}}_{e}^{\downarrow l}\left(\tilde{\mathbf{C}}_{e}^{\downarrow l}\right)^{H}\right] \Delta \mathbf{Z}_{2, l}^{H}\right\}$. Using the fact that the diagonal matrices $\Delta \mathbf{Z}_{2, l}$ and $\Delta \mathbf{Z}_{2, k}$ are independent for $l \neq k$, we can derive $\boldsymbol{\psi} \approx$ $\sum_{l=0}^{L-1}\left\|\rho_{l}\right\|^{2} \varpi \operatorname{diag}\left[\overline{\mathbf{C}}_{e}^{\downarrow l}\left(\overline{\mathbf{C}}_{e}^{\downarrow l}\right)^{H}+\tilde{\mathbf{C}}_{e}^{\downarrow l}\left(\tilde{\mathbf{C}}_{e}^{\downarrow l}\right)^{H}\right]$, substituting it into (84) leads to

$\gamma_{f d, m}=\frac{\varrho^{2}}{4} \Re\left\{\mathbf{W}_{m} \boldsymbol{\varphi}_{m}^{H} \mathbf{F}\left(\overline{\mathbf{C}}_{m}^{H} \boldsymbol{\psi} \overline{\mathbf{C}}_{m}+\tilde{\mathbf{C}}_{m}^{H} \boldsymbol{\psi} \tilde{\mathbf{C}}_{m}\right) \mathbf{F}^{H} \boldsymbol{\varphi}_{m} \mathbf{W}_{m}^{H}\right\}$

By taking the $n$-th diagonal element of $\gamma_{f d, m}$, we obtain (65).

\section{APPENDIX E}

ProOF OF EQ. (68)

Both $\mathbf{u}_{t d, m}$ and $\mathbf{u}_{f m, m}$ are a mixture function of two sets of uncorrelated random variables: $\overline{\mathbf{a}}_{m}$ and $\tilde{\mathbf{a}}_{m}$, we can write $\gamma_{f m+t d, m, n}$ in the following form

$$
\gamma_{f m+t d, m, n}=\frac{\left|W_{m, n}\right|^{2} \varrho^{2}}{2}\left(\bar{\alpha}_{f m+t d, m, n}+\tilde{\alpha}_{f m+t d, m, n}\right)
$$

where $\bar{\alpha}_{f m+t d, m, n}$ and $\tilde{\alpha}_{f m+t d, m, n}$ are due to $\overline{\mathbf{a}}_{m}$ and $\tilde{\mathbf{a}}_{m}$, respectively. Let us first consider the terms that contains $\overline{\mathbf{a}}_{m}$ only. With the definition of $\mathbf{u}_{t d, m}$ and $\mathbf{u}_{f m, m}$ in Eq. (56), we have

$$
\begin{aligned}
& \bar{\alpha}_{f m+t d, m, n}=\frac{2}{\left|W_{m, n}\right|^{2} \varrho^{2}} \mathcal{E} \| \Re\left(\sum_{i=0}^{M-1} W_{m, n} \overline{\overline{\mathbf{q}}}_{m, i, n} \Delta \mathbf{H}_{m, n} \overline{\mathbf{a}}_{i}\right) \\
& +j \Im\left(\sum_{i=0}^{M-1} W_{m, n} \overline{\tilde{\mathbf{q}}}_{m, i, n} \Delta \mathbf{H}_{m, n} \overline{\mathbf{a}}_{i}\right) \\
& +\Re\left(W_{m, n} \mathbf{f}_{n} \overline{\mathbf{C}}_{m}^{H} \sum_{l=0}^{L-1} \rho_{l} \Delta \overline{\mathbf{C}}^{\downarrow l} \overline{\mathbf{a}}_{m}\right) \\
& +j \Im\left(W_{m, n} \mathbf{f}_{n} \tilde{\mathbf{C}}_{m}^{H} \sum_{l=0}^{L-1} \rho_{l} \Delta \overline{\mathbf{C}}^{\downarrow l} \overline{\mathbf{a}}_{m}\right) \|_{n}^{2}
\end{aligned}
$$

Using $\overline{\mathbf{T}}=\sum_{l=0}^{L-1} \rho_{l} \Delta \overline{\mathbf{C}}^{\downarrow l}, \mathcal{E}\left\{\overline{\mathbf{a}}_{m}\right\}=\mathcal{E}\left\{\tilde{\mathbf{a}}_{m}\right\}=\varrho^{2} / 2$, and $W_{m, n}=\left|W_{m, n}\right| e^{j \theta_{m, n}}$ into (87), yields

$$
\begin{aligned}
& \bar{\alpha}_{f m+t d, m, n}=\| \Re\left[e ^ { j \theta _ { m , n } } \left(\sum_{i=0}^{M-1} \overline{\overline{\mathbf{q}}}_{m, i, n} \Delta \mathbf{H}_{m, n} \mathbf{f}_{n} \overline{\mathbf{C}}_{m}^{H}\right.\right. \\
& \left.\left.\sum_{l=0}^{L-1} \rho_{l} \Delta \overline{\mathbf{C}}^{\downarrow l}\right)\right]\left\|_{n}^{2}+\right\| \Im\left[e ^ { j \theta _ { m , n } } \left(\sum_{i=0}^{M-1} \overline{\tilde{\mathbf{q}}}_{m, i, n} \Delta \mathbf{H}_{m, n}\right.\right. \\
& \left.\left.+\mathbf{f}_{n} \tilde{\mathbf{C}}_{m}^{H}+\sum_{l=0}^{L-1} \rho_{l} \Delta \overline{\mathbf{C}}^{\downarrow l}\right)\right] \|_{n}^{2}
\end{aligned}
$$

Similarly, can derive $\tilde{\alpha}_{f m+t d, m, n}$ as shown in (70), substituting them into (86) leads to (68).

\section{REFERENCES}

[1] 5GNOW, "D3.1: 5G waveform candidate selection," Tech. Rep., 2013.

[2] B. Farhang-Boroujeny, "OFDM versus filter bank multicarrier," IEEE Signal Processing Magazine, vol. 28, no. 3, pp. 92-112, May 2011.

[3] F. Schaich and T. Wild, "Waveform contenders for 5G; OFDM vs. FBMC vs. UFMC," in IEEE International Symposium on Communications, Control and Signal Processing (ISCCSP), May 2014, pp. 457-460. 
[4] G. Wunder, P. Jung, M. Kasparick, T. Wild, F. Schaich, Y. Chen, S. Brink, I. Gaspar, N. Michailow, A. Festag, L. Mendes, N. Cassiau, D. Ktenas, M. Dryjanski, S. Pietrzyk, B. Eged, P. Vago, and F. Wiedmann, "5GNOW: non-orthogonal, asynchronous waveforms for future mobile applications," IEEE Communications Magazine, vol. 52, no. 2, pp. 97-105, February 2014.

[5] R. Razavi, P. Xiao, and R. Tafazolli, "Information theoretic analysis of OFDM/OQAM with utilized intrinsic interference," IEEE Signal Processing Letters, vol. 22, no. 5, pp. 618-622, May 2015.

[6] M. Schellmann, Z. Zhao, H. Lin, P. Siohan, N. Rajatheva, V. Luecken, and A. Ishaque, "FBMC-based air interface for $5 \mathrm{G}$ mobile: Challenges and proposed solutions," in IEEE International Conference on Cognitive Radio Oriented Wireless Networks and Communications (CROWNCOM), June 2014, pp. 102-107.

[7] N. Cassiau, D. Ktenas, and J.-B. Dore, "Time and frequency synchronization for CoMP with FBMC," in IEEE Proceedings of the Tenth International Symposium on Wireless Communication Systems (ISWCS 2013), Aug 2013, pp. 1-5.

[8] D. Mattera, M. Tanda, and M. Bellanger, "Analysis of an FBMC/OQAM scheme for asynchronous access in wireless communications," EURASIP Journal on Advances in Signal Processing, vol. 2015, no. 1, p. 23, 2015.

[9] Q. Bai and J. Nossek, "On the effects of carrier frequency offset on cyclic prefix based OFDM and filter bank based multicarrier systems," in IEEE Eleventh International Workshop on Signal Processing Advances in Wireless Communications (SPAWC), June 2010, pp. 1-5.

[10] H. Lin, M. Gharba, and P. Siohan, "Impact of time and carrier frequency offsets on the FBMC/OQAM modulation scheme," Signal Process, vol. 102, no. 9, pp. 151-162, 2014.

[11] Y. Cheng, P. Li, and M. Haardt, "Coordinated beamforming for the multi-user MIMO downlink using FBMC/OQAM," in IEEE International Symposium on Communications, Control and Signal Processing (ISCCSP), May 2014, pp. 465-469.

[12] P. Siohan, C. Siclet, and N. Lacaille, "Analysis and design of OFDM/OQAM systems based on filterbank theory," IEEE Transactions on Signal Processing, vol. 50, no. 5, pp. 1170-1183, May 2002.

[13] F. Schaich, "Filterbank based multi carrier transmission (fbmc); evolving OFDM: FBMC in the context of WiMAX," in Wireless Conference (EW), 2010 European, April 2010, pp. 1051-1058.

[14] I. Estella, A. Pascual-Iserte, and M. Payaro, "OFDM and FBMC performance comparison for multistream MIMO systems," in Future Network and Mobile Summit, June 2010, pp. 1-8.

[15] J. Du, P. Xiao, J. Wu, and Q. Chen, "Design of isotropic orthogonal transform algorithm-based multicarrier systems with blind channel estimation," IET Communications, vol. 6, pp. 2695-2704, November 2012.

[16] A. Ikhlef and J. Louveaux, "An enhanced MMSE per subchannel equalizer for highly frequency selective channels for FBMC/OQAM systems," in IEEE 10th Workshop on Signal Processing Advances in Wireless Communications, June 2009, pp. 186-190.

[17] T. Ihalainen, A. Ikhlef, J. Louveaux, and M. Renfors, "Channel equalization for multi-antenna FBMC/OQAM receivers," IEEE Transactions on Vehicular Technology, vol. 60, no. 5, pp. 2070-2085, Jun 2011.

[18] B. Chang, C. da Rocha, D. Le Ruyet, and D. Roviras, "On the effect of ISI in the error performance of precoded FBMC/OQAM systems," in Asia-Pacific Conference on Communications (APCC), Oct 2012, pp. 987-991.

[19] M. Soysa, N. Rajatheva, and M. Latva-Aho, "Precoding and receiver processing for multiple access MIMO FBMC systems," in Proceedings of European Wireless 2014; 20th European Wireless Conference, May 2014, pp. 1-7.

[20] M. Soysa, N. Rajatheva, and M. Latva-aho, "Linear and non-linear transceiver processing for MEVIO-FBMC systems," in IEEE International Conference on Communications (ICC), June 2014, pp. 4607-4612.

[21] Y. Zeng, Y.-C. Liang, M. W. Chia, and E. C. Y. Peh, "Unified structure and parallel algorithms for FBMC transmitter and receiver," in IEEE 24th International Symposium on Personal Indoor and Mobile Radio Communications (PIMRC), Sept 2013, pp. 922-926.

[22] B. Farhang-Boroujeny and C. G. Yuen, "Cosine modulated and offset QAM filter bank multicarrier techniques: A continuous-time prospect," EURASIP Journal on Advances in Signal Processing, vol. 2010, 2010.

[23] M. K. Simon and M.-S. Alouini, Digital Communication over Fading Channels, 2nd ed. Wiley, 2005.

[24] L. Zhang, A. U. Quddus, E. Katranaras, D. Wubben, Y. Qi, and R. Tafazolli, "Performance analysis and optimal cooperative cluster size for randomly distributed small cells under cloud RAN," IEEE Access, vol. 4, pp. 1925-1939, 2016.

[25] D. Palomar, M. Bengtsson, and B. Ottersten, "Minimum BER linear transceivers for MIMO channels via primal decomposition," IEEE
Transactions on Signal Processing, vol. 53, no. 8, pp. 2866-2882, Aug 2005.

[26] L. Zhang, P. Xiao, and A. Quddus, "Cyclic prefix-based universal filtered multicarrier system and performance analysis," IEEE Signal Processing Letters, vol. 23, no. 9, pp. 1197-1201, Sept 2016.

[27] D. S. Sesia, M. M. Baker, and M. I. Toufik, LTE: The UMTS Long Term Evolution: from Theory to Practice, 2nd ed. Wiley-Blackwell, 2011.

[28] E. Dahlman, S. Parkvall, and J. Skold, 4G: LTE/LTE-Advanced for Mobile Broadband. Academic Press, 2011.

[29] B. OHara and A. Petrick, IEEE 802.11 Handbook: A Designer's Companion, 2nd ed. IEEE, 2005.

[30] B. Picinbono and P. Chevalier, "Widely linear estimation with complex data," IEEE Transactions on Signal Processing, vol. 43, no. 8, pp. 20302033, Aug 1995. 